Algebraic $8 \mathcal{G}$ Geometric Topology

Volume 3 (2003) 473-509

ATG

Published: 5 June 2003

\title{
Transfer and complex oriented cohomology rings
}

\author{
MALKhaZ BAKURADZE \\ STEWART PRIDDY
}

\begin{abstract}
For finite coverings we elucidate the interaction between transferred Chern classes and Chern classes of transferred bundles. This involves computing the ring structure for the complex oriented cohomology of various homotopy orbit spaces. In turn these results provide universal examples for computing the stable Euler classes (i.e. $\left.\operatorname{Tr}^{*}(1)\right)$ and transferred Chern classes for $p$-fold covers. Applications to the classifying spaces of $p$-groups are given.
\end{abstract}

AMS Classification 55N22; 55R 12

Keywords Transfer, Chern class, classifying space, complex cobordism, Morava K-theory

\section{Introduction}

For various examples of finite groups the complex oriented cohomology ring coincides with its subring generated by Chern classes [21],[22], [24]. Even more groups are good in the sense that their Morava $K$-theory is generated by transferred Chern classes of complex representations of subgroups [10]. Special effort was needed to find an example of a group not good in this sense [14]. Thus the relations in the complex oriented cohomology ring of a finite group derived from formal properties of the transfer should play a major role. The purpose of this paper is to elucidate for finite coverings the interaction between transferred Chern classes and Chern classes of transferred bundles.

Let $p$ be a prime and let $G \leq \Sigma_{p}$ be a subgroup of the symmetric group. In this paper we consider the complex oriented cohomology of homotopy orbit spaces $X_{h G}^{p}=E G \times{ }_{G} X^{p}$. Several authors have computed these cohomology groups, [15], [11], [12], [10], however we are particularly interested in the ring structure and thereby explicit formulas for the transfer. Thus we are led to consider Fibrins reciprocity, the relation between cup products and transfer:

$$
\operatorname{Tr}^{*}(x) y=\operatorname{Tr}^{*}\left(x \rho^{*}(y)\right)
$$


(formula (i) of Section 2) where $\rho: E G \times X^{p} \rightarrow X_{h G}^{p}$ is the covering projection and

$$
\operatorname{Tr}^{*}: E^{*}\left(X^{p}\right) \rightarrow E^{*}\left(X_{h G}^{p}\right)
$$

is the associated transfer homomorphism.

Let $\pi \leq \Sigma_{p}$ be the subgroup of cyclic permutations of order $p$. Our results for $M U^{*}\left(X_{h \pi}^{p}\right), X=\mathbf{C} P^{\infty}$, and $\xi \rightarrow \mathbf{C} P^{\infty}$ the canonical complex line bundle, provide a universal example which enables us to write explicitly the Chern classes $c_{1}, \ldots, c_{p-1}$ of the transferred bundle $\xi_{\pi}$ as certain formal power series in the Euler class $c_{p}\left(\xi_{\pi}\right)$ with coefficients in $E^{*}(B \pi)$ plus certain transferred classes of the bundle $\xi$. In Section 3 we give an algorithm for computing these coefficients.

In paricular for $E=B P$, Brown-Peterson cohomology, the coefficients of this formal power series are invariant under the action of the normalizer of $\pi$ in $\Sigma_{p}$. This enables us to give the similar results for $\Sigma_{p}$ coverings. Moreover in Section 4 we compute the algebra $B P^{*}\left(X_{h \Sigma_{p}}^{p}\right)$ and show that its multiplicative structure is completely determined by Frobenius reciprocity.

In addition for $E=K(s)$, Morava $K$-theory, the computations become easier: we show in Section 5 that the formal power series in the algorithm above descend to polynomials. We derive an alternative way for calculation and give some examples.

Section 6 is devoted to extending some results of [10] in Morava $K$-theory. In particular we show that if $X$ is good then $X_{h \pi}^{p}$ is good.

In Section 7 we give some applications to classifying spaces of finite groups.

We would like to thank several people for their help during the course of this work: D. Ravenel for supplying us with the proof of Lemma 5.3, M. Jibladze for some Maple programs used in the examples of Section 5, and finally the referee who suggested many improvements.

The first author was supported by CRDF grant GM1 2083 and by the MaxPlanck-Institut für Mathematik.

A word about notation: in Sections $2,3,4$ and 7 we denote $\mathbf{C} P^{\infty}$ simply by $X$.

\section{Preliminaries}

We recall that a multiplicative cohomology theory $E^{*}$ is called complex oriented if there exists a Thom class, that is, a class $u \in E^{2}\left(\mathbf{C} P^{\infty}\right)$ that restricts to a 
generator of the free one-dimensional $E^{*}$ module $E^{2}\left(\mathbf{C} P^{1}\right)$. The universal example is complex cobordism $M U^{*}$. Then

$$
E^{*}\left(\mathbf{C} P^{\infty}\right)=E^{*}[[x]],
$$

where $x$ is the Euler class of the canonical complex line bundle $\xi$ over $\mathbf{C} P^{\infty}=$ $B U(1)$. Further

$$
E^{*}\left(B U(1)^{p}\right)=E^{*}\left[\left[x_{1}, \ldots, x_{p}\right]\right],
$$

where $x_{i}=c_{1}\left(\xi_{i}\right)$ and $\xi_{i}$ is the pullback bundle over $B U(1)^{p}$ by the projection $B U(1)^{p} \rightarrow B U(1)$ on the $i$-th factor.

Much of our paper is written in terms of transfer maps $[1,13]$ and formal group laws. Let us give a brief review of formal properties of the transfer. For a finite covering

$$
\rho: X \rightarrow X / G
$$

there is a stable transfer map

$$
\operatorname{Tr}=\operatorname{Tr}(\rho): X / G^{+} \rightarrow X^{+} .
$$

For any multiplicative cohomology theory $E^{*}$, Frobenius reciprocity holds i.e., the induced map $T r^{*}$ is a map of $E^{*}(X / G)$ modules

(i) $\operatorname{Tr}^{*}\left(x \rho^{*}(y)\right)=T r^{*}(x) y, x \in E^{*}(X), y \in E^{*}(X / G)$.

For example

(ii) $\operatorname{Tr}^{*}\left(\rho^{*}(y)\right)=\operatorname{Tr}^{*}(1) y$.

The element $\operatorname{Tr}^{*}(1) \in E^{0}(X / G)$ is called the index or stable Euler class of the covering $\rho$. The following additional properties of the transfer will be used:

(iii) The transfer is natural with respect to pullbacks;

(iv) $\operatorname{Tr}\left(\rho_{1} \times \rho_{2}\right)=\operatorname{Tr}\left(\rho_{1}\right) \wedge \operatorname{Tr}\left(\rho_{2}\right)$;

(v) If $\rho=\rho_{2} \rho_{1}$, then $\operatorname{Tr}(\rho)=\operatorname{Tr}\left(\rho_{2}\right) \operatorname{Tr}\left(\rho_{1}\right)$.

More generally for a covering projection

$$
\rho_{H, G}: X / H \rightarrow X / G
$$

with $H \leq G$ there is a stable transfer map

$$
\operatorname{Tr}_{H, G}: X / G^{+} \rightarrow X / H^{+} .
$$

To ease notation if $H=e$, as above, we write projection and transfer in equivalent ways $\rho=\rho_{G}, \operatorname{Tr}=\operatorname{Tr}(\rho)=\operatorname{Tr}_{G}$.

The reverse composition to (ii) is given by: 
(vi) (Double coset formula) If $K, H \leq G$ then

$$
\rho_{K, G}^{*} \operatorname{Tr}_{H, G}^{*}=\sum_{x} \operatorname{Tr}_{K \cap H^{x}, K}^{*} \circ x^{-1^{*}} \circ \rho_{K^{x^{-1}} \cap H, H}^{*}
$$

where the sum is taken over a set of double coset representatives $x \in K \backslash G / H$. Here $H^{x}=x H x^{-1}$.

For a regular covering $\rho_{H, G}$, i.e. $H \unlhd G$,

$$
\rho_{H, G}^{*} \operatorname{Tr}_{H, G}^{*}(x)=N(x)=\sum_{g \in G / H} g^{*}(x),
$$

where $N(x)$ is called the norm or trace of $x$.

In subsequent sections the reduced transfer $\operatorname{Tr}_{H, G}: X / G \rightarrow X / H$ is used.

We recall Quillen's formula [16, 6]. First,

$$
E^{*}(B \mathbf{Z} / p)=E^{*}[[z]] /([p](z)),
$$

where $x$ is the Euler class of a faithful one-dimensional complex representation of $\mathbf{Z} / p$ and $[p](z)$ is the $p$-series or $p$-fold iterated formal sum. Then

$$
\operatorname{Tr}_{\mathbf{Z} / p}^{*}(1)=[p](z) / z,
$$

where $\operatorname{Tr}_{\mathbf{Z} / p}^{*}$ is the transfer homomorphism for the universal $\mathbf{Z} / p$-covering $E \mathbf{Z} / p \rightarrow B \mathbf{Z} / p$. The relation $[p](z)=0$ is equivalent to the transfer relation

$$
z \operatorname{Tr}_{\mathbf{Z} / p}^{*}(1)=\operatorname{Tr}_{\mathbf{Z} / p}^{*}\left(c_{1}(\mathbf{C})\right)=\operatorname{Tr}_{\mathbf{Z} / p}^{*}(0)=0
$$

obtained by applying (ii). Of course since the transfer is natural, Quillen's formula enables us to compute the stable Euler class for any regular $\mathbf{Z} / p$ covering.

In this spirit, let

$$
\pi=\langle t\rangle \leq \Sigma_{p}
$$

be the subgroup of cyclic permutations of order $p$. For a given free action of $\pi$ on a space $Y$ with a given complex line bundle $\eta \rightarrow Y$ we have an equivariant map

$$
\eta_{\pi}=\left(g_{1}, \ldots, g_{p}\right): Y \rightarrow B U(1)^{p},
$$

where $g_{i}$ classifies the line bundle $t^{i-1} \eta$.

So by naturality of the transfer, the computation of transferred Chern classes $\operatorname{Tr}^{*}\left(c_{1}^{i}(\eta)\right), i \geq 1$ for cyclic coverings can be reduced to the covering

$$
\rho_{\pi}: E \pi \times(B U(1))^{p} \rightarrow E \pi \times_{\pi}(B U(1))^{p},
$$

as the universal example. 
Similarly for the symmetric group.

Let $\xi$ be the canonical complex line bundle over $\mathbf{C} P^{\infty}=B U(1)$ and $\xi_{i}$ be the pullback bundle over $B U(1)^{p}$ by the projection on the $i$-th factor as before. Then $M U^{*}\left(B U(1)^{p}\right)=M U^{*}\left[\left[x_{1}, \ldots, x_{p}\right]\right], x_{i}=c_{1}\left(\xi_{i}\right)$ and $x_{1} \cdots x_{p}$ is the Euler class of the bundle $\xi^{\times p}=\oplus \xi_{i}$.

Note that by transfer property $(\mathrm{v}), \operatorname{Tr}\left(\rho_{\pi}\right)^{*}$ has the same value on the Chern classes $x_{1}, \ldots, x_{p}$ : the group $\pi$ permutes the $x_{i}$ and $\rho_{\pi} t=\rho_{\pi}, t \in \pi$. Thus in computations of the transfer we sometimes write these Chern classes in an equivalent way $x, t x, \ldots, t^{p-1} x$.

For the sphere bundle $S\left(\xi^{\times p}\right)$, one has

$$
M U^{*}\left(S\left(\xi^{\times p}\right)\right)=M U^{*}\left[\left[x_{1}, \ldots, x_{p}\right]\right] /\left(x_{1} \cdots x_{p}\right) .
$$

Then for the trace map

$$
N=1+t+\cdots+t^{p-1}
$$

we have $\operatorname{ker} N=\operatorname{Im}(1-t), t \in \pi$ in $M U^{*} B U(1)^{p}$ and after restricting $N$ to $M U^{*}\left(S\left(\xi^{\times p}\right)\right)$ we have the exact sequence

$\cdots \leftarrow M U^{*}\left(S\left(\xi^{\times p}\right)\right) \stackrel{N}{\leftarrow} M U^{*}\left(S\left(\xi^{\times p}\right)\right) \stackrel{1-t}{\leftarrow} M U^{*}\left(S\left(\xi^{\times p}\right)\right) \stackrel{N}{\leftarrow} M U^{*}\left(S\left(\xi^{\times p}\right)\right) \leftarrow \cdots$

Then let $\xi_{\pi}=E \pi \times_{\pi} \xi^{\times p}$ be the Atiyah transfer bundle [2],

$$
S\left(\xi_{\pi}\right)=E \pi \times_{\pi} S\left(\xi^{\times p}\right)
$$

be its sphere bundle and

$$
D\left(\xi_{\pi}\right)=E \pi \times_{\pi} D\left(\xi^{\times p}\right)
$$

be its disk bundle. Let $X=\mathbf{C} P^{\infty}$ then $D\left(\xi_{\pi}\right)$ is homotopy equivalent to $X_{h \pi}^{p}=B(\pi \imath U(1))$.

The cofibration $D\left(\xi_{\pi}\right) / S\left(\xi_{\pi}\right)=\left(X_{h \pi}^{p}\right)^{\xi_{\pi}}$ gives a long exact sequence

$$
\cdots \leftarrow M U^{*}\left(S\left(\xi_{\pi}\right)\right) \leftarrow M U^{*}\left(X_{h \pi}^{p}\right) \stackrel{\times c_{p}}{\longleftarrow} M U^{*}\left(\left(X_{h \pi}^{p}\right)^{\xi_{\pi}}\right) \leftarrow \cdots
$$

where $\left(X_{h \pi}^{p}\right)^{\xi_{\pi}}$ is the Thom space of the bundle $\xi_{\pi}$ and the right homomorphism is multiplication by the Euler class $c_{p}=c_{p}\left(\xi_{\pi}\right)$.

Since the diagonal of $B U(1)^{p}$ is fixed under the permutation action of $\pi$, the inclusion $E \pi \rightarrow E \pi \times B U(1)^{p} ; x \rightarrow(x$, fixpoint $)$ defines the inclusions

$$
\begin{gathered}
i: B \pi \rightarrow X_{h \pi}^{p} \\
i_{0}: B \pi \rightarrow S\left(\xi_{\pi}\right) .
\end{gathered}
$$

Algebraic 83 Geometric Topology, Volume 3 (2003) 
The projection $\varphi: X_{h \pi}^{p} \rightarrow B \pi$ induced by $\pi \curlywedge U(1) \rightarrow \pi$ defines the projection

$$
\varphi_{0}: S\left(\xi_{\pi}\right) \rightarrow B \pi
$$

and the compositions $\varphi_{0} i_{0}, \varphi i$ are the identity. We can consider $S\left(\xi_{\pi}\right)$ as a bundle over $B \pi$ with fiber $S\left(\xi^{\times p}\right)$.

Let $\eta$ be the canonical line bundle over $B \pi$ and

$$
\theta=\varphi^{*}(\eta) \rightarrow X_{h \pi}^{p}
$$

be the pullback bundle. Thus $i^{*}(\theta)=\eta$ and $i^{*}\left(\xi_{\pi}\right)=\mathbf{C}+\eta+\cdots+\eta^{p-1}$.

Consider the pullback diagram

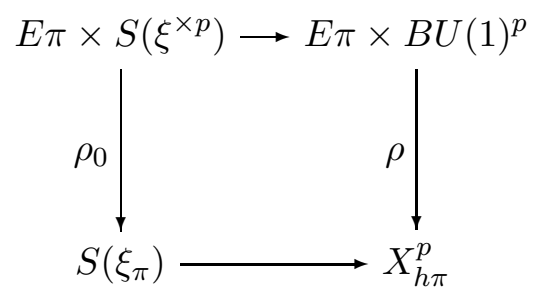

Let $\operatorname{Tr}=\operatorname{Tr}(\rho)$ be the transfer of the covering $\rho$, and $\operatorname{Tr}_{0}: S\left(\xi_{\pi}\right) \rightarrow S\left(\xi^{\times p}\right)$ the transfer map of $\rho_{0}$.

We will often refer to the following lemma which follows from (3) and Frobenius reciprocity.

Lemma 2.1 In $M U^{*}\left(X_{h \pi}^{p}\right), \operatorname{Im} \operatorname{Tr}^{*} \bigcap \operatorname{Ker}\left(\rho^{*}\right)=0$.

Proof $\rho^{*}\left(\operatorname{Tr}^{*}(a)\right)=N(a)=0 \Rightarrow a \in \operatorname{Im}(1-t) \Rightarrow \operatorname{Tr}^{*}(a)=0$.

Remark 2.2 Lemma 2.1 is valid only in complex oriented cohomology $E^{*}$ with torsion free coefficient ring. This lemma is used in the proof of Theorem 3.1 in complex cobordism and in the second statement of Theorem 4.6 in BrownPeterson cohomology. By naturality, these results hold for all $E^{*}$ in the first case and all $p$-local $E^{*}$ in the second.

\section{Transferred Chern classes for cyclic coverings}

In this section we prove our main result for cyclic coverings, Theorem 3.2.

In the notation of the previous section the $k$-th Chern class of the bundle $\xi^{\times p}$ is the elementary symmetric function $\sigma_{k}\left(x_{1}, \ldots, x_{p}\right)$ in Chern classes $x_{i}$ and 
is the sum of $\left(\begin{array}{l}p \\ k\end{array}\right)$ elementary monomials. The action of $\pi$ on the set of these monomials gives us $p^{-1}\left(\begin{array}{l}p \\ k\end{array}\right)$ orbits and the transfer homomorphism is constant on orbits by transfer property (v) or (iii).

Let $E^{*}$ be a complex oriented cohomology theory. For $k=1, \ldots, p-1$, let

$$
\omega_{k}=\omega_{k}\left(x_{1}, \ldots, x_{p}\right) \in E^{*}\left(B U(1)^{p}\right)
$$

be the sum of representative monomials one from each of these orbits. The value of $\operatorname{Tr}^{*}\left(\omega_{k}\right)$ does not depend on the choice of $\omega_{k}$ since $\omega_{k}$ is defined modulo $\operatorname{Im}(1-t)$ and on the elements of $\operatorname{Im}(1-t)$ the transfer homomorphism is zero again by (v). In other words we can take any $\omega_{k}$ for which $N \omega_{k}=\sigma_{k}\left(x_{1}, \ldots, x_{p}\right)$ holds. As we shall explain in Corollary 3.6 of Theorem 3.1, the following result enables us to calculate the transfer on all elements whose norm is symmetric.

For ease of notation let $X=\mathbf{C} P^{\infty}$ and $c_{j}=c_{j}\left(\xi_{\pi}\right), j=1, \ldots, p$.

Theorem 3.1 We can construct explicit elements

$$
\delta_{i}^{(k)} \in \tilde{E}^{*}(B \pi), k=1, \ldots, p-1,
$$

such that

$$
\operatorname{Tr}^{*}\left(\omega_{k}\right)=c_{k}+\sum_{i \geq 0} \varphi^{*}\left(\delta_{i}^{(k)}\right) c_{p}^{i}
$$

for the transfer of the covering $\rho: X^{p} \rightarrow X_{h \pi}^{p}$.

Before constructing the elements $\delta_{i}^{(k)}$ in Section 3.2 we first prove their existence.

\subsection{Complex cobordism of $\left(\mathbf{C} P^{\infty}\right)_{h \pi}^{p}$}

Theorem 3.2 In $M U^{*}\left(X_{h \pi}^{p}\right)$

(a) The annihilator of the Chern class $c=c_{1}(\theta)$ coincides with $\operatorname{ImTr}^{*}$;

(b) Multiplication by $c_{p}=c_{p}\left(\xi_{\pi}\right)$ is a monomorphism;

(c) Any element of $\operatorname{Ker}\left(\rho^{*}\right)$ has the form $\sum_{k \geq 0} \varphi^{*}\left(\delta_{k}\right) c_{p}^{k}$, for some elements $\delta_{k} \in \tilde{M} U^{*}(B \pi)$.

(d) For $\pi=\mathbf{Z} / 2$,

$$
\begin{gathered}
M U^{*} B(\pi \prec U(1))=M U^{*}\left[\left[c, c_{1}, c_{2}\right]\right] /\left(c_{1}-c_{1}^{*}, c_{2}-c_{2}^{*}\right) \\
=M U^{*}(B \pi)\left[\left[\operatorname{Tr}^{*}(x), c_{2}\right]\right] /\left(c \operatorname{Tr}^{*}(x)\right),
\end{gathered}
$$

where $c_{i}=c_{i}\left(\xi_{\pi}\right), c_{i}^{*}=c_{i}\left(\xi_{\pi} \otimes_{\mathbf{C}} \theta\right)$, and $x$ are Chern characteristic classes with $x \in M U^{*}\left(B U(1)^{2}\right)=M U^{*}[[x, t x]]$. 
We need the following lemma.

Lemma 3.3 The left homomorphism in the long exact sequence (6) is an epimorphism and thus gives a short exact sequence

$$
0 \leftarrow M U^{*}\left(S\left(\xi_{\pi}\right)\right) \leftarrow M U^{*}\left(X_{h \pi}^{p}\right) \stackrel{\times c_{p}}{\leftarrow} M U^{*}\left(X_{h \pi}^{p}\right)^{\xi_{\pi}} \leftarrow 0 .
$$

Moreover there is a space $X_{\pi}$ and a stable equivalence

$$
\varphi_{0} \vee f_{\pi}: S\left(\xi_{\pi}\right) \rightarrow B \pi \vee X_{\pi},
$$

with $f_{\pi}$ factoring through the following composite map

$$
S\left(\xi_{\pi}\right) \rightarrow X_{h \pi}^{p} \stackrel{T r}{\rightarrow} E \pi \times B U(1)^{p}
$$

and $\varphi_{0}$ as in (7).

Proof Consider the Serre spectral sequence for the fibration (7)

$$
S\left(\xi^{\times p}\right) \rightarrow S\left(\xi_{\pi}\right) \stackrel{\varphi_{0}}{\longrightarrow} B \pi .
$$

$E_{2}^{i, j}=H^{i}\left(\pi, H^{j}\left(S\left(\xi^{\times p}\right) ; \mathbf{F}_{q}\right)\right)$ with the action of $\pi$ on $H^{*}\left(S\left(\xi^{\times p}\right) ; \mathbf{F}_{q}\right)$ by permutations of the cohomological Chern classes.

When $q=p, E_{2}^{0, j}=H^{j}\left(S\left(\xi^{\times p}\right) ; \mathbf{F}_{p}\right)^{\pi}$ and $E_{2}^{i, 0}=H^{i}\left(B \pi ; \mathbf{F}_{p}\right)$.

Then in positive dimensions $H^{*}\left(S\left(\xi^{\times p}\right) ; \mathbf{F}_{q}\right)=\mathbf{F}_{q}\left[x_{1}, \ldots, x_{p}\right] /\left(x_{1} \cdots x_{p}\right)$ is a permutation representation of $\pi$ acting on monomials which have degree zero in at least one indeterminate. This is a free $\mathbf{F}_{q}[\pi]$-module since all the monomials that are fixed under this action have been factored out after quotienting by the ideal $\left(x_{1} \cdots x_{p}\right)$. Hence the cohomology of $\pi$ with coefficients in this module is trivial in positive dimensions, i.e. $E_{2}^{i, j}=0$ when $i, j>0$. Thus the spectral sequence collapses and we have

$$
H^{*}\left(S\left(\xi_{\pi}\right) ; \mathbf{F}_{p}\right) \approx H^{*}\left(B \pi ; \mathbf{F}_{p}\right) \oplus \tilde{H}^{*}\left(S\left(\xi^{\times p}\right) ; \mathbf{F}_{p}\right)^{\pi} .
$$

Also if $q \neq p$ we have $H^{*}\left(S\left(\xi_{\pi}\right) ; \mathbf{F}_{q}\right) \approx H^{*}\left(S\left(\xi^{\times p}\right) ; \mathbf{F}_{q}\right)^{\pi}$.

Let $X_{\pi}$ be a stable summand of $B U(1)^{p}$ defined as follows. The action of $\pi$ on $B U(1)^{p}$ induces an action of $\pi$ on the stable decomposition of $B U(1)^{p}$ as a wedge of all smash products of length $1, \ldots, p-1$, say $Y_{\pi}$, and a smash product of length $p$. Then choose $X_{\pi}$ such that $N X_{\pi}=Y_{\pi}$, where $N=1+t+\cdots+t^{p-1}$. By the stable equivalence

$$
S\left(\xi^{\times p}\right) \rightarrow B U(1)^{p} \rightarrow Y_{\pi}
$$


we can consider $X_{\pi}$ as a stable summand of $S\left(\xi^{\times p}\right)$. For any choice of $X_{\pi}$, consider the composition of stable maps

$$
f_{\pi}: S\left(\xi_{\pi}\right) \rightarrow X_{h \pi}^{p} \stackrel{T r}{\rightarrow} E \pi \times B U(1)^{p} \rightarrow B U(1)^{p} \rightarrow X_{\pi} .
$$

We have to show that the stable map $\varphi_{0} \vee f_{\pi}$ induces an isomorphism in cohomology for any group of coefficients $\mathbf{F}_{q}, q$ a prime, and hence gives a stable equivalence by the stable Whitehead lemma. It follows from the above arguments that

$$
\tilde{H}^{*}\left(S\left(\xi_{\pi}\right) ; \mathbf{F}_{p}\right)=\varphi_{0}^{*} \tilde{H}^{*}\left(B \pi ; \mathbf{F}_{p}\right) \oplus \operatorname{Tr}_{0}^{*} \tilde{H}^{*}\left(S\left(\xi^{\times p}\right) ; \mathbf{F}_{p}\right),
$$

and $\tilde{H}^{*}\left(S\left(\xi_{\pi}\right) ; \mathbf{F}_{q}\right)=T r_{0}^{*} \tilde{H}^{*}\left(S\left(\xi^{\times p}\right) ; \mathbf{F}_{q}\right)$, when $q \neq p$. The restriction of $\operatorname{Tr}_{0}$

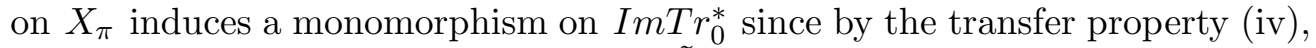
$\rho_{0}^{*} \operatorname{Tr}_{0}^{*}=N$ and the restriction of $N$ on $\tilde{H}^{*}\left(X_{\pi} ; \mathbf{F}_{q}\right)$ is a monomorphism. Hence $\left(\varphi_{0} \vee \operatorname{Tr}_{0} \mid X_{\pi}\right)^{*}$ is an isomorphism and so is $\left(\varphi_{0} \vee f_{\pi}\right)^{*}$ by the commutative diagram

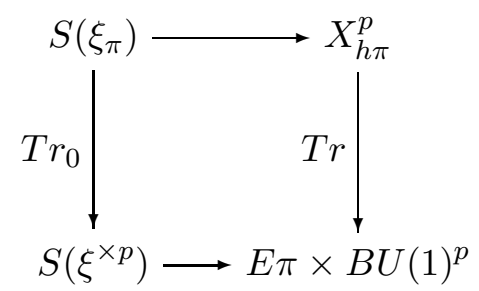

This proves Lemma 3.3 .

Proof of Theorem 3.2 (a) We consider the restriction of any element $y \in$ $M U^{*}\left(X_{h \pi}^{p}\right)$ to $M U^{*}\left(S\left(\xi_{\pi}\right)\right)$. By Lemma 3.3 we see this restriction has the form $\varphi_{0}^{*}(u)+f_{\pi}^{*}(w)$ for some $u \in \tilde{M} U^{*}(B \pi), w \in M U^{*}\left(X_{\pi}\right)$. Since the composition $S\left(\xi_{\pi}\right) \rightarrow X_{h \pi}^{p} \stackrel{\varphi}{\rightarrow} B \pi$ coincides with $\varphi_{0}, \varphi^{*}(u)$ also restricts to $\varphi_{0}^{*}(u)$. By diagram (10) there is an element $v \in M U^{*}\left(B U(1)^{p}\right)$ such that $\operatorname{Tr}^{*}(v)$ restricts to $f_{\pi}^{*}(w)$. By exactness

$$
y=\varphi^{*}(u)+\operatorname{Tr}^{*}(v)+y_{1} c_{p},
$$

for some $y_{1} \in M U^{*}\left(X_{h \pi}^{p}\right)$. For use in the proof of (c) we observe that (2), (8) imply $v$ can be chosen in the direct summand $M U^{*}\left[\left[x_{1}, \ldots, x_{p}\right]\right] /\left(x_{1} \cdots x_{p}\right)$. Thus we can assume this expression for $y$ is unique and if $v \neq 0$ then $\operatorname{Tr}^{*}(v)$ restricts nontrivally in $M U^{*}\left(S\left(\xi_{\pi}\right)\right)$.

Then suppose $c y=0$. We know that $\rho^{*}(\theta)=\mathbf{C}$, hence $\rho^{*}(c)=0$ and $c \operatorname{Tr}^{*}(v)=\operatorname{Tr}^{*}\left(\rho^{*}(c) v\right)=0$ by Frobenius reciprocity. So we have $c \varphi^{*}(u)+$ $c y_{1} c_{p}=0$. We want to prove $\varphi^{*}(u) \in \operatorname{ImTr^{*}}$. Applying $i^{*}$ we have $0=$ $i^{*}\left(c \varphi^{*}(u)\right)+i^{*}\left(c y_{1} c_{p}\right)=z u$ since $i^{*} \varphi^{*}=i d, c=\varphi^{*}(z)$, and $i^{*}\left(c_{p}\right)=0$. Hence 
$u \in \operatorname{Ann}(z)=\operatorname{Im}_{\operatorname{Tr}}^{*} \mathbf{Z}_{/ p}$. By naturality of the transfer $\varphi^{*}(u) \in \operatorname{Im} \operatorname{Tr}^{*}$. Thus $c \varphi^{*}(u)=0$ and therefore $c y_{1} c_{p}=0$. Multiplication by $c_{p}$ is injective by Lemma 3.3 , hence $c y_{1}=0$. Since $\operatorname{dim}\left(y_{1}\right)=\operatorname{dim}(y)-2 p$ iterating this argument gives us statement (a).

(b) This follows from the fact that the right homomorphism in the short exact sequence from Lemma 3.3 is multiplication by the Euler class $c_{p}\left(\xi_{\pi}\right)$.

(c) Let $y \in K \operatorname{Ker} \rho^{*}$. Since $\varphi \rho=*$ we have

$$
0=\rho^{*}\left(\operatorname{Tr}^{*}(v)\right)+\rho^{*}\left(y_{1} c_{p}\right) .
$$

If the first summand is not zero it restricts nontrivially in $M U^{*}\left(S\left(\xi^{\times p}\right)\right)$ by definition of $v$. However the second summand restricts to zero since $\rho^{*}\left(c_{p}\right)=$ $x_{1} \cdots x_{p}, \quad \rho^{*}\left(y_{1} c_{p}\right)=\rho^{*}\left(y_{1}\right) x_{1} \cdots x_{p}$ and $x_{1} \cdots x_{p}$ restricts to zero as the Euler class. Hence both summands are zero. Furthermore multiplication by $x_{1} \cdots x_{p}$ is a monomorphism hence $\rho^{*}\left(y_{1}\right)=0$. So

$$
y=\varphi^{*}(u)+y_{1} c_{p}=\varphi^{*}(u)+\left(\varphi^{*}\left(u_{1}\right)+y_{2} c_{p}\right) c_{p}=\varphi^{*}(u)+\varphi^{*}\left(u_{1}\right) c_{p}+y_{2} c_{p}^{2} .
$$

Repetition of this process proves (c).

(d) The fact that $c, c_{1}, c_{2}$ multiplicatively generate $M U^{*} B(\pi \imath U(1))$ follows from Lemma 3.3. The relations $c_{1}=c_{1}^{*}, c_{2}=c_{2}^{*}$ follow from the bundle relation

$$
\xi_{\pi} \otimes_{\mathbf{C}} \theta=\left(\xi \otimes_{\mathbf{C}} \rho^{*}(\theta)\right)_{\pi}=\xi_{\pi},
$$

which in turn follows from transfer property (i).

So we have to prove that the Chern classes $c, c_{1}, c_{2}$ with these relations are a complete system of generators and relations. Let us use the splitting principle to write formally

$$
\begin{gathered}
\xi_{\pi}=\eta_{1}+\eta_{2} ; \\
u_{1}=c_{1}\left(\eta_{1}\right) ; \\
u_{2}=c_{1}\left(\eta_{2}\right) .
\end{gathered}
$$

Let $F(x, y)=\sum \alpha_{i j} x^{i} y^{j}$ be the formal group law. Using the bundle relation above and applying the Whitney formula for the first and second Chern classes, we obtain two relations of the form:

$$
F\left(u_{1}, c\right)+F\left(u_{2}, c\right)=c_{1}
$$

and

$$
F\left(u_{1}, c\right) F\left(u_{2}, c\right)=c_{2}
$$

or in terms of $c, c_{1}=u_{1}+u_{2}, c_{2}=u_{1} u_{2}$

$$
F\left(u_{1}, c\right)+F\left(u_{2}, c\right)-c_{1}=c\left(2+\sum \beta_{i j k} c^{i} c_{1}^{j} c_{2}^{k}\right)=0
$$


and

$$
F\left(u_{1}, c\right) F\left(u_{2}, c\right)-c_{2}=c\left(c_{1}+\sum \gamma_{i j k} c^{i} c_{1}^{j} c_{2}^{k}\right)=0,
$$

for some coefficients $\beta_{i j k}, \gamma_{i j k} \in M U^{*}(p t)$.

We claim that relations (12) and (13) are equivalent to the following two obvious transfer relations for $T r^{*}: M U^{*}[[x, t x]] \rightarrow M U^{*}(B(\pi \imath U(1)))$

$$
c \operatorname{Tr}^{*}(1)=0
$$

and

$$
c \operatorname{Tr}^{*}(x)=0 \text {. }
$$

Rewrite relations (12) and (13) as follows:

$$
\begin{gathered}
c a=0, \quad \text { where } a=2+\alpha_{11} c_{1}+\sum_{k \geq 2} \alpha_{k 1}\left(u_{1}^{k}+u_{2}^{k}\right)+o(c), \\
c b=0, \quad \text { where } b=c_{1}+2 \alpha_{11} c_{2}+\sum_{k \geq 2} \alpha_{k 1}\left(u_{1}^{k-1}+u_{2}^{k-1}\right) c_{2}+o(c),
\end{gathered}
$$

and the $\alpha_{i j}$ are the coefficients of the formal group law.

By the first part of Theorem 3.2, $a \in \operatorname{ImTr}^{*}$. Also by transfer property (vi)

$$
\rho^{*}(a)=\rho^{*}\left(\operatorname{Tr}^{*}(1)+\alpha_{11} \operatorname{Tr}^{*}(x)+\sum_{k \geq 2} \alpha_{k 1} \operatorname{Tr}^{*}\left(x^{k}\right)\right) .
$$

Thus by Lemma 2.1

$$
\operatorname{Tr}^{*}(1)+\alpha_{11} \operatorname{Tr}^{*}(x)+\sum_{k \geq 2} \alpha_{k 1} \operatorname{Tr}^{*}\left(x^{k}\right)=\left(F\left(u_{1}, c\right)+F\left(u_{2}, c\right)-c_{1}\right) / c ;
$$

similarly $b \in \operatorname{Im} T r^{*}$ and

$$
\operatorname{Tr}^{*}(x)+\alpha_{11} \operatorname{Tr}^{*}(1) c_{2}+\sum_{k \geq 2} \alpha_{k 1} \operatorname{Tr}^{*}\left(x^{k-1}\right) c_{2}=\left(F\left(u_{1}, c\right) F\left(u_{2}, c\right)-c_{2}\right) / c .
$$

Now since

$$
x^{k}=x^{k-1}(x+t x)-x^{k-2}(x t x),
$$

transfer property (i) and the computation of $\operatorname{Tr}^{*}(x)$ is sufficient for the computation of $\operatorname{Tr}^{*}\left(x^{k}\right), k \geq 2$ (see also Corollary 3.6, Remark 3.7). So we have

$$
\operatorname{Tr}^{*}(1)\left(1+g_{0}\right)+\operatorname{Tr}^{*}(x) h_{0}=\left(F\left(u_{1}, c\right)+F\left(u_{2}, c\right)-c_{1}\right) / c,
$$

and

$$
\operatorname{Tr}^{*}(1) g_{1}+\operatorname{Tr}^{*}(x)\left(1+h_{1}\right)=\left(F\left(u_{1}, c\right) F\left(u_{2}, c\right)-c_{2}\right) / c,
$$

where $g_{0}, h_{0}, g_{1}, h_{1} \in M U^{*}(B(\pi \imath U(1)))$. This proves (d).

This completes the proof of Theorem 3.2.

Algebraic 83 Geometric Topology, Volume 3 (2003) 
Formula (15) for computing $\operatorname{Tr}^{*}(x)$ is complicated; let us give a simpler form. Consider again (13). Note that the coefficient $\gamma_{00 k} \in M U^{*}(p t)$ contains a factor 2: the element

$$
c_{1}+\sum \gamma_{i j k} c^{i} c_{1}^{j} c_{2}^{k}
$$

annihilates $c$ and hence belongs to $\operatorname{ImTr^{*}}$. On the other hand

$$
\rho^{*} \operatorname{Tr}^{*}=1+t ; \rho^{*}(c)=0 ; \rho^{*}\left(c_{1}\right)=x+t x ; \rho^{*}\left(c_{2}\right)=x t x,
$$

hence applying $\rho^{*}$ we have that

$$
x+t x+\sum \gamma_{0 j k}(x+t x)^{j}(x t x)^{k}
$$

belongs to $\operatorname{Im}(1+t)$. So $\gamma_{00 k}(x t x)^{k}=2 \gamma_{k}(x t x)^{k}$, that is, $\gamma_{00 k}=2 \gamma_{k}$ for some coefficient $\gamma_{k}$.

Recall that on the other hand $F(c, c)=0$ that is $2 c=o\left(c^{2}\right)$. So $\gamma_{00 k} c=o\left(c^{2}\right)$, hence taking into account the relation $F(c, c)=0$ we can rewrite (13) after division by

$$
1+\sum_{i, k \geq 0} \gamma_{i 1 k} c^{i} c_{2}^{k}
$$

(the coefficient at $c c_{1}$ ) as follows

$$
c c_{1}=d_{0} c+d_{2} c c_{1}^{2}+\cdots+d_{n} c c_{1}^{n}+\cdots,
$$

where $d_{k}=d_{k}\left(c, c_{2}\right) \in M U^{*}\left[\left[c, c_{2}\right]\right]$ and $d_{0}\left(0, c_{2}\right)=0$; the lower index $n$ indicates the coefficient at $c c_{1}^{n}$. Since

$$
\rho^{*}\left(\operatorname{Tr}^{*}(x)-c_{1}\right)=0,
$$

it follows from Theorem 3.2(c) that there exist elements

$$
\delta_{j} \in \tilde{M} U^{*}(B \pi)
$$

such that

$$
\operatorname{Tr}^{*}(x)=c_{1}+\sum_{j \geq 0} \varphi^{*}\left(\delta_{j}\right) c_{2}^{j} .
$$

Using the inclusion $i: B \pi \rightarrow B(\pi \imath U(1))$ we have

$$
i^{*}\left(c_{1}\right)=i_{0}^{*}(c) ; i^{*} \operatorname{Tr}^{*}(x)=0 ; i^{*}\left(c_{2}\right)=0,
$$

thus

$$
\varphi^{*}\left(\delta_{0}\right)=-c .
$$

For the calculation of the other elements $\delta_{j}$ recall that $c \operatorname{Tr}^{*}(x)=0$, hence

$$
c c_{1}^{n}=-c \varphi^{*}\left(\delta^{n}\right) ; n \geq 1,
$$


where

$$
\delta=-c+\sum_{j \geq 1} \delta_{j} c_{2}^{j}
$$

Combining (16) and (17), we have the following:

Proposition 3.4 The elements $\delta_{j}, j>0$ can be determined from the recurrence relations which arise from the following formula in $M U^{*}(B \pi)\left[\left[c_{2}\right]\right]$

$$
\delta=d_{0}+\sum_{i \geq 2} d_{i} \delta^{i}
$$

Proof By definition the element $\delta-d_{0}-\sum_{i \geq 2} d_{i} \delta^{i}$ belongs to Ker $\rho^{*}$. On the other hand this element is annihilated by $c$ hence

$$
\delta-d_{0}-\sum_{i \geq 2} d_{i} \delta^{i} \in \operatorname{Im} \operatorname{Tr}^{*} \bigcap \operatorname{Ker}\left(\rho^{*}\right)=0
$$

by Lemma 2.1 .

Corollary 3.5 For the elements $\delta_{j} \in \tilde{M} U^{*}(B \mathbf{Z} / 2)$, constructed in 3.4 , the following formula holds in $M U^{*} B(\mathbf{Z} / 2 \prec U(1))$

$$
\operatorname{Tr}^{*}(x)=c_{1}-c+\sum_{j \geq 1} \varphi^{*}\left(\delta_{j}\right) c_{2}^{j}
$$

In fact we have proved Theorem 3.1 for $p=2$. The general case, analogous but more technical, is given next.

\subsection{Proof of Theorem 3.1}

Note that by the definition of $\omega_{k}$ the difference $T r^{*}\left(\omega_{k}\right)-c_{k}$ is an element of $\operatorname{Ker}\left(\rho^{*}\right)$. Thus Theorem 3.2 (c) implies existence of the elements $\delta_{i}^{(k)}$ in Theorem 3.1.

First let us elucidate the meaning of the relations

$$
\xi_{\pi} \otimes_{\mathbf{C}} \theta=\xi_{\pi}
$$

in the general case of $B(\pi<U(1))$.

Again, we can use the splitting principle and write formally

$$
\xi_{\pi}=\eta_{1}+\eta_{2}+\cdots+\eta_{p} ; u_{m}=c_{1}\left(\eta_{m}\right), m=1, \ldots, p .
$$


Applying the Whitney formula for the relation

$$
\eta_{1} \otimes_{\mathbf{C}} \theta+\cdots+\eta_{p} \otimes_{\mathbf{C}} \theta=\eta_{1}+\cdots+\eta_{p},
$$

and taking into account that $c_{m}=c_{m}\left(\xi_{\pi}\right)$ is the elementary symmetric function $\sigma_{m}\left(u_{1}, \ldots, u_{p}\right)$ we have

$$
\sigma_{m}\left(F\left(u_{1}, c\right), \ldots, F\left(u_{p}, c\right)\right)=c_{m},
$$

$m=1, \ldots, p$, or in terms of $c, c_{1}, \ldots, c_{p}$ we have

$$
c\left(p+\sum \beta_{i_{0}, i_{1}, \ldots, i_{p}}^{0} c^{i_{0}} c_{1}^{i_{1}} \cdots c_{p}^{i_{p}}\right)=0
$$

and

$$
c\left((p-k) c_{k}+\sum \beta_{i_{0}, i_{1}, \ldots, i_{p}}^{k} c^{i_{0}} c_{1}^{i_{1}} \cdots c_{p}^{i_{p}}\right)=0 ;
$$

for $k=1, \ldots, p-1$ and some $\beta_{i_{0}, i_{1}, \ldots, i_{p}}^{0}, \beta_{i_{0}, i_{1}, \ldots, i_{p}}^{k} \in M U^{*}(p t)$.

We claim that these relations are equivalent to the following obvious relations

$$
c \operatorname{Tr}^{*}(1)=0
$$

and

$$
c \operatorname{Tr}^{*}\left(\omega_{k}\right)=0,
$$

for the elements $\omega_{k} \in M U^{*}(B U(1))^{p}, k=1, \ldots, p-1$ defined above.

For the proof of our claim multiply the $k$-th relation from (19) by $p_{k}=(p-k)^{-1}$ in $\mathbf{F}_{p}$. Then by Theorem 3.2, Ann (c) coincides with $\operatorname{ImTr}{ }^{*}$ hence (18) implies that

$$
p_{k}\left(\sigma_{k+1}\left(F\left(u_{1}, c\right), \ldots, F\left(u_{p}, c\right)\right)-c_{k+1}\right) / c=\operatorname{Tr}^{*}\left(a_{k}\right),
$$

for some $a_{k}$ which we have to find. Let us write

$$
\begin{gathered}
\rho^{*}\left(p_{k}\left(\sigma_{k+1}\left(F\left(u_{1}, c\right), \ldots, F\left(u_{p}, c\right)\right)-c_{k+1}\right) / c\right)=g^{(k)}\left(\sigma_{1}, \ldots, \sigma_{p}\right) \\
=\sigma_{k}\left(1+g_{k}^{(k)}\left(\sigma_{1}, \ldots, \sigma_{p}\right)\right)+\sum_{j \neq k, 1 \leq j \leq p-1} \sigma_{j} g_{j}^{(k)}\left(\sigma_{j}, \sigma_{j+1}, \ldots, \check{\sigma_{k}}, \ldots, \sigma_{p}\right) \\
=N\left(\omega_{k}\right)\left(1+g_{k}^{(k)}\left(\sigma_{1}, \ldots, \sigma_{p}\right)\right)+\sum_{j \neq k, 1 \leq j \leq p-1} N\left(\omega_{j}\right) g_{j}^{(k)}\left(\sigma_{j}, \sigma_{j+1}, \ldots, \check{\sigma_{k}}, \ldots, \sigma_{p}\right) .
\end{gathered}
$$

Here the symbol $\check{\sigma_{k}}$ indicates absence of the corresponding term. So we have

$$
\begin{gathered}
p_{k}\left(\sigma_{k+1}\left(F\left(u_{1}, c\right), \ldots, F\left(u_{p}, c\right)\right)-c_{k+1}\right) / c \\
=\operatorname{Tr}^{*}\left(\omega_{k}\right)\left(1+g_{k}^{(k)}\left(c_{1}, \ldots, c_{p}\right)\right)+\sum_{j \neq k, 1 \leq j \leq p-1} \operatorname{Tr}^{*}\left(\omega_{j}\right) g_{j}^{(k)}\left(c_{j}, c_{j+1}, \ldots, \check{c_{k}}, \ldots, c_{p}\right),
\end{gathered}
$$

and

$$
\left[\sigma_{1}\left(F\left(u_{1}, c\right), \ldots, F\left(u_{p}, c\right)\right)-c_{1}\right] / c
$$

Algebraic 63 Geometric Topology, Volume 3 (2003) 


$$
=\operatorname{Tr}^{*}(1)\left(1+g_{0}^{(0)}\left(c_{1}, \ldots, c_{p}\right)\right)+\sum_{1 \leq j \leq p-1} \operatorname{Tr}^{*}\left(\omega_{j}\right) g_{j}^{(0)}\left(c_{j}, c_{j+1}, \ldots, c_{p}\right) .
$$

This proves our claim.

For computing $\delta_{i}^{(k)}$ we start with the equations (19) and rewrite them as

$$
c f_{k}\left(c, c_{1}, \ldots, c_{p}\right)=0, \quad k=1, \ldots, p-1 .
$$

These are equations in a power series algebra $M U^{*}(B \pi)\left[\left[c_{p}\right]\right]$, since we know $c c_{k} \in c M U^{*}(B \pi)\left[\left[c_{p}\right]\right]$.

We now want to find explicitly formal series

$$
\delta^{(k)}\left(c_{p}\right)=\sum_{i \geq 0} \delta_{i}^{(k)}(c) c_{p}^{i}
$$

such that

$$
\operatorname{Tr}^{*}\left(\omega_{k}\right)=c_{k}+\delta^{(k)}\left(c_{p}\right)
$$

and hence

$$
c c_{k}^{j}=-c\left(\delta^{(k)}\left(c_{p}\right)\right)^{j}, \quad j \geq 1 .
$$

For this we want to replace the equations (20) by the equations

$$
c \widetilde{f}_{k}\left(c, \delta^{(1)}\left(c_{p}\right), \ldots, \delta^{(p-1)}\left(c_{p}\right), c_{p}\right)=0,
$$

where $\tilde{f}_{k} \in \operatorname{Ker} \rho_{\pi}^{*}$ is a series whose coefficient at $\delta^{(k)}$ is invertible. In fact $\widetilde{f}_{k}=0$ since we know that $\operatorname{Ann}(c)=\operatorname{ImTr^{*}}$ and $\operatorname{Ker}\left(\rho^{*}\right) \cap \operatorname{ImTr^{*}}=0$ by Lemma 2.1.

Then equating each coefficient of the resulting series

$$
g_{k}\left(c_{p}\right)=\widetilde{f}_{k}\left(c, \delta^{(1)}\left(c_{p}\right), \ldots, \delta^{(p-1)}\left(c_{p}\right), c_{p}\right)=0
$$

in the ring $M U^{*}(B \pi)\left[\left[c_{p}\right]\right]$ to zero we will obtain $p-1$ infinite strings of equations in $M U^{*}(B \pi)$. Assuming $\delta_{i}^{(l)}$ are already found for $i<n$ we get

$$
\delta_{n}^{(k)}=\psi_{n, k}\left(\left(\delta_{i}^{(1)}\right)_{i \leq n}, \ldots,\left(\delta_{i}^{(k)}\right)_{i<n}, \ldots,\left(\delta_{i}^{(p-1)}\right)_{i \leq n}\right),
$$

a system of linear equations in $\delta_{n}^{(l)}, l=1, \ldots, p-1$ with invertible determinant and coefficients in $M U^{*}[[c]]$. Since the $\delta_{0}^{(l)}$ are already known as $l$-th Chern classes of the bundle $1+\theta+\cdots+\theta^{p-1}$, by induction on $n$ we can solve formally (25) to get

$$
\delta_{n}^{(k)}(c)=\tilde{\psi}_{n, k}\left(\left(\delta_{i}^{(l)}\right)_{i<n}\right) .
$$

This gives $\delta_{n}^{(k)}=\delta_{n}^{(k)}(z) \in M U^{*}(B \pi)$ obviously satisfying our equations. 
Now for the remaining equation (23) we proceed as follows: let us look at the term $c f_{k}\left(0,0, \ldots, 0, c_{p}\right)$ in equations $(20)$. Note that $f_{k}\left(0,0, \ldots, 0, c_{p}\right)$ is divisible by $p$ :

$f_{k} \in \operatorname{Ann}(c)=\operatorname{ImTr^{*}} \Rightarrow \rho_{\pi}^{*} f_{k} \in \operatorname{Im} N \Rightarrow f_{k}\left(0, \ldots, 0, \sigma_{p}\right) \in \operatorname{Im} N \Rightarrow$ $f_{k}\left(0, \ldots, 0, \sigma_{p}\right)$ is divisible by $p$.

Next using the relation $[p]_{F}(c)=0$ we know that $p c$ is divisible by $c^{2}$; hence each occurrence of $p c$ in these equations can be replaced by terms with higher powers of $c$. So $c f_{k}\left(0,0, \ldots, 0, c_{p}\right)$ can be replaced by a term divisible by $c^{2}$.

Also the $k$-th relation from (20) contains the term $c(p-k) c_{k}$, and for the condition (24) we have to multiply the $k$-th equation from $(20)$ by $(p-k)^{-1}$, the inverse of $p-k$ in $\mathbf{F}_{p}$, and as above we can replace $c(p-k) c_{k}$ by $c c_{k}+$ (terms divisible by $c^{2}$ ). Then we use (22) and substitute the series $\delta^{(k)}$ in the resulting equations, thus obtaining (23).

This completes the proof for $E=M U$, which is the universal example of complex oriented cohomology theories. From this result we can descend to all E.

We now turn to computation of $\operatorname{Tr}^{*}$ in general.

Corollary 3.6 For all primes p, Theorem 3.1 enables us to explicitly compute the transfer homomorphism for those polynomials $a \in \tilde{M U}^{*}\left[\left[x_{1}, \ldots, x_{p}\right]\right]$ for which $N a=a+t a+\cdots+t^{p-1} a$ is symmetric in $x_{1}, \ldots, x_{p}$.

Proof If $N a=\sigma_{1} a_{1}\left(\sigma_{1}, \ldots, \sigma_{p}\right)+\ldots+\sigma_{p-1} a_{p-1}\left(\sigma_{1}, \ldots, \sigma_{p}\right)$, then

$$
\left.\operatorname{Tr}^{*}(a)=\operatorname{Tr}^{*}\left(\omega_{1}\right) a_{1}\left(c_{1}, \ldots, c_{p}\right)+\cdots+\operatorname{Tr}^{*}\left(\omega_{p-1}\right) a_{p-1}\left(c_{1}, \ldots, c_{p}\right)\right) .
$$

To see this let $\hat{a}=\omega_{1} a_{1}\left(\sigma_{1}, \ldots, \sigma_{p}\right)+\cdots+\omega_{p-1} a_{p-1}\left(\sigma_{1}, \ldots, \sigma_{p}\right)$. Then $N(a-$ $\hat{a})=0$, that is, $a-\hat{a} \in \operatorname{Im}(1-t)$, hence $\operatorname{Tr}^{*}(a)=\operatorname{Tr}^{*}(\hat{a})$.

Remark 3.7 For $p=2$ one has recurrence formulas for $\operatorname{Tr}^{*}\left(x^{k}\right), k \geq 1$.

$$
\begin{gathered}
\operatorname{Tr}^{*}(x)=\operatorname{Tr}^{*}\left(\omega_{1}\right) \\
\operatorname{Tr}^{*}\left(x^{k}\right)=\operatorname{Tr}^{*}\left(x^{k-1}\right) c_{1}-\operatorname{Tr}^{*}\left(x^{k-2}\right) c_{2}
\end{gathered}
$$

This follows using the formula $x^{k}=x^{k-1}(x+t x)-x^{k-2}(x t x)$. 


\section{Transferred Chern classes for $\Sigma_{p}$-coverings}

If we consider a $p$-local complex oriented cohomology $E^{*}$ then by standard transfer arguments (see Lemma 4.3 below) $E^{*}\left(B \Sigma_{p}\right)$ is isomorphic to the subring of $E^{*}(B \pi)$ invariant under the action of the normalizer of $\pi$ in $\Sigma_{p}$. The results of this section imply the elements $\delta_{i}^{(k)} \in \tilde{E}^{*}(B \pi)$ from Theorem 3.1 are invariant under this action. This defines elements $\tilde{\delta}_{i}^{(k)} \in \tilde{E}^{*}\left(B \Sigma_{p}\right)$ which we use for computing the transfer.

In this section we consider $B P^{*}\left(X_{h \Sigma_{p}}^{p}\right)$ for $X=\mathbf{C} P^{\infty}$ and for the covering projection

$$
\rho_{\Sigma_{p}}: E \Sigma_{p} \times X^{p} \rightarrow X_{h \Sigma_{p}}^{p}
$$

we give a formula for the transfer homomorphism

$$
\operatorname{Tr}_{\Sigma_{p}}{ }^{*}: B P^{*}\left(X^{p}\right) \rightarrow B P^{*}\left(X_{h \Sigma_{p}}^{p}\right)
$$

using the elements $\tilde{\delta}_{i}^{(k)}$.

\subsection{Brown-Peterson cohomology of $\left(\mathbf{C} P^{\infty}\right)_{h \Sigma_{p}}^{p}$.}

We need definitions analogous to those of Section 2, with the cyclic group replaced by the symmetric group. The $p$-fold product, $\xi^{\times p}$, of the canonical line bundle over $X^{p}$ extends to an $p$-dimensional bundle

$$
\xi_{\Sigma_{p}}=E \Sigma_{p} \times \Sigma_{p} \xi^{\times p}
$$

over $X_{h \Sigma_{p}}^{p}$ classified by the inclusion $X_{h \Sigma_{p}}^{p}=B\left(\Sigma_{p} \imath U(1)\right) \hookrightarrow B U(p)$. Let $c_{i}=c_{i}\left(\xi_{\Sigma_{p}}\right)$. Then $\rho_{\Sigma_{p}}{ }^{*}\left(c_{i}\right)=c_{i}\left(\xi^{\times p}\right)=\sigma_{i}$, the $i$-th symmetric polynomial in the $x_{j}$, where $B P^{*}\left(X^{p}\right)=B P^{*}\left[\left[x_{1}, \ldots, x_{p}\right]\right]$.

Then we have the projection

$$
\varphi: X_{h \Sigma_{p}}^{p} \rightarrow B \Sigma_{p}
$$

induced by the factorization $\Sigma_{p} \prec U(1) / U(1)^{p}=\Sigma_{p}$ and the inclusion

$$
i: B \Sigma_{p} \rightarrow X_{h \Sigma_{p}}^{p}
$$

induced by the inclusion of $\Sigma_{p}$ in $\Sigma_{p}$ २U(1).

Definition 4.1 Let $\tilde{c}_{i}=\operatorname{Tr}_{\Sigma_{p}}{ }^{*}\left(x_{1} x_{2} \cdots x_{i}\right)$ for $i=1, \ldots, p-1$.

Lemma $4.2 \rho_{\Sigma_{p}}{ }^{*}\left(\tilde{c}_{i}\right)=i !(p-i) ! \sigma_{i}$. 
For the group $W=N_{\Sigma_{p}}(\pi) / \pi \approx \mathbf{Z} /(p-1),|W|$ is prime to $p$, hence by the standard transfer argument $\rho_{\pi, \Sigma_{p}}^{*}: B P^{*}\left(B \Sigma_{p}\right) \rightarrow B P^{*}(B \pi)$ is an injective map of $B P^{*}$ algebras. Since $\rho_{\pi, \Sigma_{p}}^{*}\left(y T r_{\Sigma_{p}}^{*}(1)\right)=p ! z^{p-1}$ plus terms of higher filtration, $y \operatorname{Tr}_{\Sigma_{p}}^{*}(1)=0$ is the only relation.

Relating $\pi$ and $\Sigma_{p}$ we have a lift of $\rho_{\pi, \Sigma_{p}}$

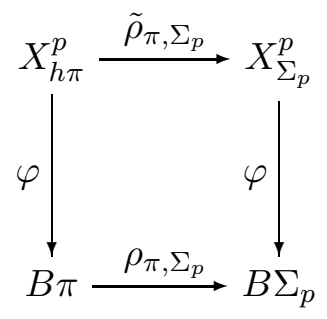

Lemma $4.4 \tilde{\rho}_{\pi, \Sigma_{p}}^{*}\left(\widetilde{c}_{k}\right)=k !(p-k) ! \operatorname{Tr}_{\pi}^{*}\left(\omega_{k}\right)$.

Proof Note that modulo $\operatorname{Im}(1-t)$ we have $\Sigma g^{*}\left(x_{1} x_{2} \cdots x_{k}\right)=k !(p-k) ! \omega_{k}$ summed over $\Sigma_{p} / \pi$. Applying the double coset formula

$$
\begin{aligned}
& \rho_{\pi, \Sigma_{p}}^{*}\left(\widetilde{c}_{k}\right)=\rho_{\pi, \Sigma_{p}}^{*} \operatorname{Tr}_{\Sigma_{p}}^{*}\left(x_{1} x_{2} \cdots x_{k}\right)= \\
& \operatorname{Tr}_{\pi}^{*} \sum_{g \in \Sigma_{p} / \pi} g^{*}\left(x_{1} x_{2} \cdots x_{k}\right)=k !(p-k) ! \operatorname{Tr}_{\pi}^{*}\left(\omega_{k}\right) .
\end{aligned}
$$

Let $c=\varphi^{*}(y) \in B P^{2(p-1)}\left(X_{h \Sigma_{p}}^{p}\right)$.

Lemma 4.5 $\operatorname{ImTr}_{\Sigma_{p}}^{*}$ is contained in the $B P^{*}$ algebra generated by

$$
c, \tilde{c}_{1}, \ldots, \tilde{c}_{p-1}, c_{p}
$$

Proof By the Künneth isomorphism,

$$
B P^{*}\left(X^{p}\right)=B P^{*}(X)^{\otimes p}=F \oplus T
$$

as a $\pi$-module, where $F$ is free and $T$ is trivial. Explicitly a $B P^{*}$ basis for $T$ is $\left\{x_{1}^{i} \cdots x_{p}^{i}, i \geq 0\right\}$, while a $B P^{*}$ basis for $F$ is $\left\{x_{1}^{i_{1}} \cdots x_{p}^{i_{p}}, i_{j} \geq 0\right\}$ where not all the exponents are equal.

By Lemma $4.3 \operatorname{Tr}_{\Sigma_{p}}^{*}(1)$ is a power series in $c$. Now recall from [9], p. 44, that we can consider $B P^{*}\left(X^{p}\right), X=\mathbf{C} P^{\infty}$ as a free $B P^{*}\left[\left[\sigma_{1}, \ldots, \sigma_{p}\right]\right]$ module generated by 1 and the elements $x_{1}^{i_{1}} \cdots x_{p}^{i_{p}} \in F$, with $0 \leq i_{j} \leq p-j$. So by Frobenius reciprocity it suffices to compute the transfer on these monomials. 
Summed over the symmetric group $\sum g^{*}\left(x_{1}^{i_{1}} \cdots x_{p}^{i_{p}}\right)$ is a symmetric function and hence has the form

$$
\sum_{\pi} \sum_{\Sigma_{p} / \pi} g^{*}\left(x_{1}^{i_{1}} \cdots x_{p}^{i_{p}}\right)=\sigma_{1} s_{1}+\cdots+\sigma_{p-1} s_{p-1}=\sum_{\pi}\left(\omega_{1} s_{1}+\cdots+\omega_{p-1} s_{p-1}\right),
$$

for the elements $\omega_{k}$ from Theorem 3.1 and symmetric functions $s_{1}, \ldots, s_{p-1}$. Hence modulo $\operatorname{ker} N_{\pi}=\operatorname{Im}(1-t), t \in \pi$, we have the following equation in $F$

$$
\sum_{\Sigma_{p} / \pi} g^{*}\left(x_{1}^{i_{1}} \cdots x_{p}^{i_{p}}\right)=\omega_{1} s_{1}+\cdots+\omega_{p-1} s_{p-1} .
$$

The left sum consists of $(p-1)$ ! elements each having the same transfer value. Also $\omega_{k}$ is the sum of $p^{-1}\left(\begin{array}{l}p \\ k\end{array}\right)$ elements $x_{i_{1}} \cdots x_{i_{k}}$; on each of these elements the transfer evaluates to $\operatorname{Tr}_{\Sigma_{p}}^{*}\left(x_{1} \cdots x_{k}\right)=\tilde{c}_{k}$. Thus Frobenius reciprocity and Lemma 4.2 is all that is needed for computing $\operatorname{Tr}_{\Sigma_{p}}^{*}$.

Recall the elements $\delta_{i}^{(k)} \in \tilde{B P^{*}}(B \pi)$ derived from Theorem 3.1 by naturality. By the standard transfer argument again the map induced by $\tilde{\rho}_{\pi, \Sigma_{p}}: X_{h \pi}^{p} \rightarrow$ $X_{h \Sigma_{p}}^{p}$, the lift of $\rho_{\pi, \Sigma_{p}}: B \pi \rightarrow B \Sigma_{p}$, is also injective. Moreover for $B P^{*}\left(X_{h \Sigma_{p}}^{p}\right)$ the ring structure is completely determined by the following:

Theorem 4.6 As a $B P^{*}$ algebra

$$
B P^{*}\left(X_{h \Sigma_{p}}^{p}\right)=B P^{*}\left[\left[c, \tilde{c}_{1}, \ldots, \tilde{c}_{p-1}, c_{p}\right]\right] /\left(c \operatorname{Tr}_{\Sigma_{p}}^{*}(1), c \tilde{c}_{i}\right)
$$

and one has the formula

$$
\widetilde{c}_{k}-k !(p-k) ! c_{k}=\Sigma_{i \geq 0} \varphi^{*}\left(\tilde{\delta}_{i}^{(k)}\right) c_{p}^{i}, \quad k=1, \ldots, p-1,
$$

where the elements $\tilde{\delta}_{i}^{(k)} \in \tilde{B P^{*}}\left(B \Sigma_{p}\right)$ are determined by

$$
\rho_{\pi, \Sigma_{p}}^{*}\left(\tilde{\delta}_{j}^{(k)}\right)=k !(p-k) ! \delta_{j}^{(k)}, \quad j \geq 0 .
$$

For the proof we follow that of Theorem 3.2. Let

$$
S\left(\xi_{\Sigma_{p}}\right)=E \Sigma_{p} \times_{\Sigma_{p}} S\left(\xi^{\times p}\right)
$$

be the sphere bundle of the bundle $\xi_{\Sigma_{p}}$ of (28). $X_{h \Sigma_{p}}^{p}$ is homotopy equivalent to the disk bundle $D\left(\xi_{\Sigma_{p}}\right)=E \Sigma_{p} \times \Sigma_{p} D\left(\xi^{\times p}\right)$. Then we have the obvious inclusion $i_{0}: B \Sigma_{p} \rightarrow S\left(\xi_{\Sigma_{p}}\right)$ and projection $\varphi_{0}: S\left(\xi_{\Sigma_{p}}\right) \rightarrow B \Sigma_{p}$ with fiber $S\left(\xi^{\times p}\right) . \varphi_{0} i_{0}$ is the identity. Thus stably $B \Sigma_{p}$ is a wedge summand of $S\left(\xi_{\Sigma_{p}}\right)$. As for the other summand let

$$
X_{\Sigma_{p}}=\vee_{i=1}^{p-1} E \Sigma_{i} \times_{\Sigma_{i}} B U(1)^{\wedge i} .
$$

By the standard transfer argument, localized at $p, X_{\Sigma_{p}}$ is a stable summand of $\vee_{i=1}^{p-1} E \Sigma_{i} \times B U(1)^{\wedge i}$ and hence of $E \Sigma_{p} \times B U(1)^{\times p}$. From this we derive the following result. 
Lemma 4.7 One has a stable equivalence localized at $p$

$$
\varphi_{0} \vee f_{\Sigma_{p}}: S\left(\xi_{\Sigma_{p}}\right) \rightarrow B \Sigma_{p} \vee X_{\Sigma_{p}}
$$

with $f_{\Sigma_{p}}$, the composition of stable maps

$$
f_{\Sigma_{p}}: S\left(\xi_{\Sigma_{p}}\right) \rightarrow X_{h \Sigma_{p}}^{p} \stackrel{T r_{\Sigma_{p}}}{\rightarrow} E \Sigma_{p} \times B U(1)^{p} \rightarrow X_{\Sigma_{p}}
$$

Proof The inclusion $i_{0}$ splits off $\varphi_{0}^{*} H^{*}\left(B \Sigma_{p}\right)$ in $H^{*}\left(S\left(\xi_{\Sigma_{p}}\right)\right)$. Furthermore in mod- $p$ cohomology

$$
H^{*}\left(S\left(\xi^{\times p}\right)\right)=\mathbf{F}_{p}\left[x_{1}, \ldots, x_{p}\right] /\left(\sigma_{p}\right),
$$

hence

$$
H^{*}\left(S\left(\xi^{\times p}\right)\right)^{\Sigma_{p}} \approx \mathbf{F}_{p}\left[\tilde{c}_{1}, \ldots, \tilde{c}_{p-1}\right]
$$

by Lemma 4.2 .

Then $H:=\tilde{H}^{*}\left(S\left(\xi^{\times p}\right)\right)$ is a free $\pi$ module and $H^{*}\left(\Sigma_{p} ; H\right) \subseteq H^{*}(\pi ; H)$. Thus

$$
\begin{aligned}
H^{*}\left(\Sigma_{p} ; H\right) & =H^{\Sigma_{p}} & \text { if } & *=0 \\
& =0 & \text { if } & *>0 .
\end{aligned}
$$

Therefore there is an isomorphism

$$
H^{*}\left(S\left(\xi_{\Sigma_{p}}\right)\right) \stackrel{\rho^{*} \oplus i_{0}^{*}}{\longrightarrow} \tilde{H}^{*}\left(S\left(\xi^{\times p}\right)\right)^{\Sigma_{p}} \oplus H^{*}\left(B \Sigma_{p}\right)
$$

where $\rho: S\left(\xi^{\times p}\right) \rightarrow S\left(\xi_{\Sigma_{p}}\right)$ is the projection. We have to prove that the first summand is $f_{\Sigma_{p}}^{*} \tilde{H}^{*}\left(X_{\Sigma_{p}}\right)$.

By naturality of the transfer we have the commutative diagram

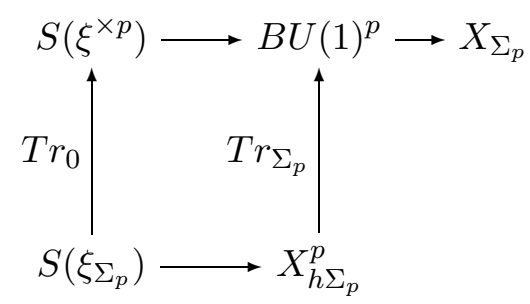

Thus $f_{\Sigma_{p}}$ coincides with $\tilde{f}_{\Sigma_{p}}$, the map $T r_{0}$ followed by the horizontal maps in the above diagram. We wish to show the restriction of $T r_{0}^{*}$ to the image of $H^{*}\left(X_{\Sigma_{p}}\right)$ is an isomorphism onto $H^{*}\left(S\left(\xi^{\times p}\right)\right)^{\Sigma_{p}}$.

Now considering the transfer for the $\Sigma_{i}$ coverings

$$
E \Sigma_{i} \times B U(1)^{\wedge i} \rightarrow E \Sigma_{i} \times_{\Sigma_{i}} B U(1)^{\wedge i}
$$


it follows from transfer properties (ii) and (vi) that $H^{*}\left(E \Sigma_{i} \times_{\Sigma_{i}} B U(1)^{\wedge i}\right)$ is a submodule of $H^{*}\left(E \Sigma_{i} \times B U(1)^{\wedge i}\right)$ generated by $\Sigma_{i}$ norms of monomials in $x_{1}, x_{2}, \ldots, x_{i}$, with non-increasing degrees. From this it is straightforward that $H^{*}\left(X_{\Sigma_{p}}\right)$ and $H^{*}\left(S\left(\xi^{\times p}\right)\right)^{\Sigma_{p}}$ have the same ranks in each dimension. Thus we are reduced to showing the desired map is injective.

However, for any monomial $x$ in $x_{1}, x_{2}, \ldots, x_{i}$, we have

$$
\operatorname{Tr}_{0}^{*}\left(N_{\Sigma_{i}}(x)\right)=i ! T r_{0}^{*}(x)
$$

by naturality of the transfer. Thus the restriction of $T r_{0}^{*}$ to the image of $H^{*}\left(X_{\Sigma_{p}}\right)$ will be a monomorphism if $\operatorname{Tr}_{0}^{*}$ is non-zero on polynomials consisting of monomials with non-increasing degrees. This in turn will follow if the norm $N_{\Sigma_{p}}$ is non-zero on such polynomials. In fact we claim: 1) $N_{\Sigma_{p}}$ is non-zero on any monomial $x^{I}=x_{1}^{i_{1}} \cdots x_{p-1}^{i_{p-1}}$, and 2) different monomials with nonincreasing degrees in $x_{1}, \ldots, x_{i}, i<p$ are in different $\Sigma_{p}$ orbits.

Claim 2) is clear. To see 1$)$ let $J=\left(j_{1}, \ldots, j_{p}\right)$ and $x^{J}=x_{1}^{j_{1}} \cdots x_{p}^{j_{p}}$, all of whose exponents are not equal. Then we will show the coefficient of $x^{J}$ in $N_{\Sigma_{p}}\left(x^{J}\right)$ is prime to $p$. The isotropy subgroup of $x^{J}$ is the finite product $\Sigma_{n_{1}} \times \Sigma_{n_{2}} \times \cdots<\Sigma_{p}$ where $n_{j}$ is the number of terms of $J$ equaling $j$. This group has order $n_{1} ! n_{2} ! \cdots$ which is prime to $p$. Hence $N_{\Sigma_{p}}(x)=\left(n_{1} ! n_{2} ! \cdots\right) x^{J}$ + other monomials proving the claim.

Thus $\varphi_{0} \vee f_{\Sigma_{p}}$ induces an isomorphism and hence is a $p$-local stable equivalence.

This implies the following:

Lemma 4.8 The long exact sequence for the pair $\left(D\left(\xi_{\Sigma_{p}}\right), S\left(\xi_{\Sigma_{p}}\right)\right)$ gives the following short exact sequence

$$
0 \leftarrow B P^{*}\left(S\left(\xi_{\Sigma_{p}}\right)\right) \leftarrow B P^{*}\left(X_{h \Sigma_{p}}^{p}\right) \leftarrow B P^{*}\left(\left(X_{h \Sigma_{p}}^{p}\right)^{\xi_{\Sigma_{p}}}\right) \leftarrow 0 .
$$

Indeed the left arrow is an epimorphism by Lemma 4.7 and hence the right arrow is a monomorphism.

Now the proof of Theorem 4.6 is completely analogous to that of Theorem 3.2 taking into account additionally that any element $y \in B P^{*}\left(X_{h \pi}^{p}\right)$ has the form

$$
y=\varphi^{*}(u)+g\left(\tilde{c}_{1}, \ldots, \tilde{c}_{p-1}\right)+y_{1} c_{p}
$$

for some $u \in B P^{*}\left(X_{h \pi}^{p}\right)$, where $g$ denotes some formal power series and $y_{1} \in$ $B P^{*}\left(X_{h \pi}^{p}\right)$. This follows by Lemma 4.5 and Lemma 4.8. 


\section{Calculation of the elements $\delta_{i}^{(k)}$ and $\tilde{\delta}_{i}^{(k)}$ in Morava $K$-theory}

In this section we work in Morava $K$-theory $K(s)^{*}$ and give an alternative, better algorithm for explicit computations.

Fix a prime $p$ and an integer $s \geq 0$, then $K(s)^{*}=\mathbf{F}_{p}\left[v_{s}, v_{s}^{-1}\right]$ with $\left|v_{s}\right|=$ $-2\left(p^{s}-1\right)$. By a result of Würgler [23] there is no restriction on $p$ : although $K(s)$ is not a commutative ring spectrum for $p=2$, we shall consider only those spaces whose Morava $K$-theory is even dimensional. This implies the deviation from commutativity is zero.

We recall

$$
K(s)^{*}(B \pi)=K(s)^{*}[z] /\left(z^{p^{s}}\right)
$$

where $|z|=2$.

As in Lemma 4.3. we have:

Lemma 5.1 (i) $\rho_{\pi, \Sigma_{p}}: B \pi \rightarrow B \Sigma_{p}$ induces an isomorphism of $K(s)^{*}$ algebras

$$
\rho_{\pi, \Sigma_{p}}^{*}: K(s)^{*}\left(B \Sigma_{p}\right) \stackrel{\approx}{\longrightarrow}\left\{K(s)^{*}(B \pi)\right\}^{W},
$$

where $W=N_{\Sigma_{p}}(\pi) / \pi \approx \mathbf{Z} /(p-1)$. Computing invariants yields

$$
K(s)^{*}\left(B \Sigma_{p}\right)=K(s)^{*}[y] /\left(y^{m_{s}}\right),
$$

where $\rho_{\pi, \Sigma_{p}}^{*}(y)=z^{p-1}$ and $m_{s}=\left[\left(p^{s}-1\right) /(p-1)\right]+1$.

(ii) $\operatorname{Tr}_{\Sigma_{p}}^{*}(1)=-v_{s} y^{m_{s}-1}$.

Then combining Theorem 4.6 and Remark 2.2 we have

$$
K(s)^{*}\left(X_{h \Sigma_{p}}^{p}\right)=K(s)^{*}\left[\left[c, \tilde{c}_{1}, \ldots, \tilde{c}_{p-1}, c_{p}\right]\right] /\left(c^{m_{s}}, c \tilde{c}_{i}\right) .
$$

Our main result in this section is the following:

Proposition 5.2 We can construct explicit elements $\delta_{i}^{(k)} \in K(s)^{*}(B \pi)$ such that

(1) In $K(s)^{*}\left(X_{h \pi}^{p}\right)$ the following formula holds

$$
c_{k}\left(\xi_{\pi}\right)=T r_{\pi}^{*}\left(\omega_{k}\right)-\sum_{0 \leq i \leq p^{s}} \varphi_{\pi}^{*}\left(\delta_{i}^{(k)}\right) c_{p}^{i}\left(\xi_{\pi}\right) .
$$

Algebraic 63 Geometric Topology, Volume 3 (2003) 
(2) In $K(s)^{*}\left(X_{h \Sigma_{p}}^{p}\right)$ one has

$$
c_{k}\left(\xi_{\Sigma_{p}}\right)=\operatorname{Tr}_{\Sigma_{p}}^{*}\left(x_{1} \cdots x_{k}\right)-\sum_{0 \leq i \leq p^{s}} \varphi_{\Sigma_{p}}^{*}\left(\tilde{\delta}_{i}^{(k)}\right) c_{p}^{i}\left(\xi_{\Sigma_{p}}\right),
$$

with $\tilde{\rho}_{\pi, \Sigma_{p}}^{*}\left(\tilde{\delta}_{i}^{(k)}\right)=k !(p-k) ! \delta_{i}^{(k)} k$.

(3) The value of $\operatorname{Tr}_{\pi}^{*}\left(c_{1}\left(\xi_{i}\right)\right)$ is determined by

$$
c_{1}\left(\xi_{\pi}\right)=\operatorname{Tr}_{\pi}^{*}\left(c_{1}\left(\xi_{i}\right)\right)+v_{s} \sum_{1 \leq j \leq s-1} c^{p^{s}-p^{j}} c_{p}^{p^{j-1}}\left(\xi_{\pi}\right)
$$

where $\xi_{i}$ is the pullback of the canonical line bundle $\xi$ by projection $B U(1)^{p} \rightarrow$ $B U(1)$ on the $i$-th factor.

We are grateful to D. Ravenel for supplying us with the proof of the following result.

Lemma 5.3 For the formal group law in Morava $K$-theory $K(s), s>1$, we have

$$
F(x, y) \equiv x+y-v_{s} \sum_{0<j<p} p^{-1}\left(\begin{array}{l}
p \\
j
\end{array}\right)\left(x^{p^{s-1}}\right)^{j}\left(y^{p^{s-1}}\right)^{p-j}
$$

modulo $x^{p^{2(s-1)}}$ (or modulo $y^{p^{2(s-1)}}$ ).

Proof This result can be derived from the recursive formula for the FGL given in 4.3.9 [17]. For the FGL in Morava $K$-theory it reads

$$
F(x, y)=\sum_{i \geq 0}^{F} v_{s}^{e_{i}} w_{i}(x, y)^{p^{i(s-1)}}
$$

where $w_{i}$ is a certain homogeneous polynomial of degree $p^{i}$ defined by 4.3 .5 [17] and $e_{i}=\left(p^{i s}-1\right) /\left(p^{s}-1\right)$. In particular $\omega_{0}=x+y$,

$$
w_{1}=-\sum_{0<j<p} p^{-1}\left(\begin{array}{l}
p \\
j
\end{array}\right) x^{j} y^{p-j}
$$

and $w_{i} \notin\left(x^{p}, y^{p}\right)$.

We find it more convenient to express $F(x, y)$ as

$$
F(x, y)=F\left(x+y, v_{s} w_{1}(x, y)^{p^{s-1}}, v_{s}^{e_{2}} w_{2}(x, y)^{p^{2(s-1)}}, \ldots\right) .
$$

Algebraic 63 Geometric Topology, Volume 3 (2003) 
Then for $s>1$ we can reduce modulo the ideal $v_{s}^{e_{2}}\left(x^{p^{2(s-1)}}, y^{p^{2(s-1)}}\right)$ and get

$$
\begin{aligned}
F(x, y) & \equiv F\left(x+y, v_{s} w_{1}(x, y)^{p^{s-1}}\right) \\
& =F\left(x+y+v_{s} w_{1}(x, y)^{p^{s-1}}, v_{s} w_{1}\left(x+y, v_{s} w_{1}(x, y)^{p^{s-1}}\right)^{p^{s-1}}, \ldots\right) \\
& \equiv F\left(x+y+v_{s} w_{1}(x, y)^{p^{s-1}}, v_{s} w_{1}\left(x^{p^{s-1}}+y^{p^{s-1}}, v_{s}^{p^{s-1}} w_{1}(x, y)^{p^{2(s-1)}}\right)\right),
\end{aligned}
$$

and modulo $v_{s}^{1+p^{s-1}}\left(x^{p^{2(s-1)}}, y^{p^{2(s-1)}}\right)$ we have

$$
F(x, y) \equiv x+y+v_{s} w_{1}(x, y)^{p^{s-1}} .
$$

Let us write for short $\sigma_{k}=\sigma_{k}(x, F(x, z), \ldots, F(x,(p-1) z))$.

Corollary 5.4 The following formula holds in $K(s)^{*}(B U(1) \times B \pi)$

$$
\sigma_{k}=-\sum_{0 \leq i \leq p^{s}} \lambda_{i}^{(k)} \sigma_{p}^{i}+p^{-1}\left(\begin{array}{l}
p \\
k
\end{array}\right) x^{k} v_{s} z^{p^{s}-1},
$$

where $\lambda_{i}^{(k)}=\lambda_{i}^{(k)}\left(z^{p-1}\right)$ are polynomials in $z^{p-1}$ and $\lambda_{0}^{(j)}=0, j=1, \ldots, p-2$, $\lambda_{0}^{(p-1)}=-z^{p-1}$.

Proof. For $1 \leq k \leq p-1$, equating the coefficients of $x^{i p}, 1 \leq i \leq p^{s}$ gives a system of linear equations with invertible matrix of the form $I d+$ nilpotent. Thus the elements $\lambda_{1}^{(k)}, \ldots, \lambda_{p^{s}}^{(k)}$ can be defined as the solution of this system. Of course equating the coefficients at $x^{i}$ for $i \neq p, 2 p, \ldots, p^{s+1}$ will produce other equations in $\lambda_{j}^{(k)}, j=1, \ldots, p^{s}$. But these equations are derived from the old equations above. These additional equations make the matrix upper triangular.

Now, let us prove Proposition 5.2 and show that one necessarily has $\delta_{i}^{(k)}=\lambda_{i}^{(k)}$, $i=0, \ldots, p^{s}$ for $\lambda_{i}^{(k)}$ encountered in Corollary 5.4. Thus by Lemma 5.1, $\delta_{i}^{(k)}$ is invariant under the action of $W$ and we can define $\tilde{\delta}_{i}^{(k)}$ by $\tilde{\rho}_{\pi, \Sigma_{p}}^{*}\left(\tilde{\delta}_{i}^{(k)}\right)=$ $k !(p-k) ! \delta_{i}^{(k)}$.

The diagonal map $\Delta: B U(1) \rightarrow B U(1)^{p}$ induces an inclusion $B \pi \times B U(1) \rightarrow$ $X_{h \pi}^{p}$ and the commutative diagram

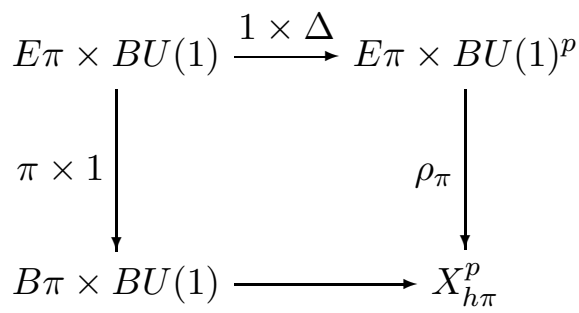

Algebraic 63 Geometric Topology, Volume 3 (2003) 
Then $(1 \times \Delta)^{*}\left(\omega_{k}\right)=p^{-1}\left(\begin{array}{l}p \\ k\end{array}\right) x^{k}, x=c_{1}(\xi)$. Hence by transfer properties (i) and (iv) we have for the transfer $\operatorname{Tr}=\operatorname{Tr}(\pi \times 1)$ :

$$
\operatorname{Tr}^{*}\left((1 \times \Delta)^{*}\left(\omega_{k}\right)\right)=p^{-1}\left(\begin{array}{l}
p \\
k
\end{array}\right) x^{k} \operatorname{Tr}^{*}(1)=p^{-1}\left(\begin{array}{l}
p \\
k
\end{array}\right) x^{k} v_{s} z^{p^{s}-1} .
$$

On the other hand by the existence of the elements $\delta_{i}^{(k)}$ (Theorem 3.1) we have

$$
\begin{gathered}
\operatorname{Tr}^{*}\left((1 \times \Delta)^{*}\left(\omega_{k}\right)\right)= \\
\sigma_{k}(x, F(x, z), \ldots, F(x,(p-1) z))+\sum_{i \geq 0} \delta_{i}^{(k)} \sigma_{p}^{i}(x, F(x, z), \ldots, F(x,(p-1) z)):
\end{gathered}
$$

$\xi_{\pi}$ restricts to $\sum_{i} \xi \otimes \theta^{i}$ on $B U(1) \times B \pi$, thus $c_{k}\left(\xi_{\pi}\right)$ to $\sigma_{k}(x, F(x, z), \ldots$, $F(x,(p-1) z))$; by Lemma 5.3 and the fact that $z^{p^{s}}=0,[i] z$ may be replaced by $i z$. By Corollary 5.4

$$
\begin{gathered}
\sigma_{k}(x, F(x, z), \ldots, F(x,(p-1) z))= \\
-\sum_{0 \leq i \leq p^{s}} \lambda_{i}^{(k)} \sigma_{p}^{i}(x, F(x, z), \ldots, F(x,(p-1) z))+p^{-1}\left(\begin{array}{l}
p \\
k
\end{array}\right) x^{k} v_{s} z^{p^{s}-1} .
\end{gathered}
$$

Then the restriction of $(1 \times \Delta)^{*}$ to $K e r \rho^{*}$ is a monomorphism [11]. This proves Proposition 5.2.1) and shows $\delta_{i}^{(k)}=\lambda_{i}^{(k)}$ for $0 \leq i \leq p^{s}$ and zero otherwise. Statement 2) follows from Lemma 4.4. Then 3) follows from the following explicit formula for $\sigma_{1}$ :

Lemma 5.5 In $K(s)^{*}(B \pi \times B U(1))$ one has

$$
\sigma_{1}=v_{s}\left(z^{p^{s}-1} x+\sum_{i=1}^{s-1} z^{p^{s}-p^{i}} \sigma_{p}^{p^{i-1}}\right) .
$$

Proof One has

$$
\begin{aligned}
\sigma_{1}=x+F(x, z)+\cdots+F(x,(p-1) z)=x+x+z+v_{s} w_{1}\left(x^{p^{s-1}}, z^{p^{s-1}}\right) \\
+\cdots+x+(p-1) z+v_{s} w_{1}\left(x^{p^{s-1}},((p-1) z)^{p^{s-1}}\right) \\
=p x+\frac{p(p-1)}{2} z+v_{s}\left(\sum_{i=1}^{p-1} w_{1}(x, i z)\right)^{p^{s-1}} \\
=v_{s}\left(\sum_{i=1}^{p-1} \sum_{j=1}^{p-1}-p^{-1}\left(\begin{array}{c}
p \\
j
\end{array}\right) i^{j} x^{p-j} z^{j}\right)^{p^{s-1}} \\
=v_{s}\left(\sum_{j=1}^{p-1}-\left(\sum_{i=1}^{p-1} i^{j}\right) p^{-1}\left(\begin{array}{c}
p \\
j
\end{array}\right) z^{j} x^{p-j}\right)^{p^{s-1}} .
\end{aligned}
$$

Algebraic 83 Geometric Topology, Volume 3 (2003) 
Now $\sum_{i=1}^{p-1} i^{j}$ is an integral linear combination of $\sigma_{k}(1,2, \ldots, p-1)$ with $k \leq i$, hence by it is zero for $i<p-1$ and for $i=p-1$ it is $p-1$.

Thus

$$
\sigma_{1}=-v_{s}\left((p-1) p^{-1}\left(\begin{array}{c}
p \\
p-1
\end{array}\right) z^{p-1} x^{p-(p-1)}\right)^{p^{s-1}}=v_{s} z^{p^{s}-p^{s-1}} x^{p^{s-1}}
$$

ow since $F(x, z)^{p}=x^{p}+z^{p}$, one has $\sigma_{p}^{p}=(x(x+z) \cdots(x+(p-1) z))^{p}$.

But again we have $x(x+z) \cdots(x+(p-1) z)=x^{p}-x z^{p-1}$. Substituting this one obtains

$$
\begin{aligned}
v_{s}\left(z^{p^{s}-1} x\right. & \left.+\sum_{i=1}^{s-1} z^{p^{s}-p^{i}} \sigma_{p}^{p^{i-1}}\right) \\
& =v_{s}\left(z^{p^{s}-1} x+z^{p^{s}-p} \sigma_{p}+\sum_{i=2}^{s-1} z^{p^{s}-p^{i}}\left(x^{p}-z^{p-1} x\right)^{p^{i-1}}\right) \\
& =v_{s}\left(z^{p^{s}-1} x+z^{p^{s}-p} \sigma_{p}+\sum_{i=2}^{s-1} z^{p^{s}-p^{i}}\left(x^{p^{i}}-z^{(p-1) p^{i-1}} x^{p^{i-1}}\right)\right)
\end{aligned}
$$

But it is straightforward to see that

$$
\sum_{i=2}^{s-1} z^{p^{s}-p^{i}}\left(x^{p^{i}}-z^{(p-1) p^{i-1}} x^{p^{i-1}}\right)=z^{p^{s}-p^{s-1}} x^{p^{s-1}}-z^{p^{s}-p} x^{p} .
$$

Hence one has

$$
\begin{aligned}
v_{s}\left(z^{p^{s}-1} x\right. & \left.+\sum_{i=1}^{s-1} z^{p^{s}-p^{i}} \sigma_{p}^{p^{i-1}}\right) \\
& =v_{s}\left(z^{p^{s}-1} x+z^{p^{s}-p} \sigma_{p}+z^{p^{s}-p^{s-1}} x^{p^{s-1}}-z^{p^{s}-p} x^{p}\right)
\end{aligned}
$$

Now one has

$z^{p^{s}-p} F(x, k z)=z^{p^{s}-p}\left(x+k z+v_{s} w_{1}\left(x^{p^{s-1}},(k z)^{p^{s-1}}\right)\right)=z^{p^{s}-p}(x+k z)$,

hence $z^{p^{s}-p} \sigma_{p}=z^{p^{s}-p} x(x+z) \cdots(x+(p-1) z)=z^{p^{s}-p}\left(x^{p}-z^{p-1} x\right)$

Substituting this into (33) gives

$$
v_{s}\left(z^{p^{s}-1} x+\sum_{i=1}^{s-1} z^{p^{s}-p^{i}} \sigma_{p}^{p^{i-1}}\right)=v_{s} z^{p^{s}-p^{s-1}} x^{p^{s-1}}
$$

which is $\sigma_{1}$ by $(32)$.

Algebraic 63 Geometric Topology, Volume 3 (2003) 
We now compute some of the elements $\delta_{i}^{(k)}$ and $\tilde{\delta}_{i}^{(k)}$.

First recall from [8], [17] that generators for

$$
\begin{array}{ccc}
\pi_{*} B P & \subset & H_{*} B P \\
\uparrow & & \uparrow \\
\mathbf{Z}_{(p)}\left[v_{1}, v_{2}, \ldots\right] & \subset & \mathbf{Z}_{(p)}\left[m_{1}, m_{2}, \ldots\right] \\
\left|v_{n}\right|=2\left(p^{n}-1\right)=\left|m_{n}\right|
\end{array}
$$

are given by

$$
v_{n}=p m_{n}-\sum_{i=1}^{n-1} m_{i} v_{n-i}^{p^{i}} .
$$

Given a formal group law over a graded ring $R_{*}$,

$$
F(x, y)=\sum_{i, j} \alpha_{i j}^{R} x^{i} y^{j} \in R_{*}[[x, y]], \quad \alpha_{i j}^{R} \in R_{2(i+j-1)}
$$

there is a ring map $g: M U_{*} \longrightarrow R_{*}$ which induces the formal group law; that is $g^{*}\left(\alpha_{i j}^{M U}\right)=\alpha_{i j}^{R}$.

We use also the following well known formulas

$$
F(x, y)=\exp (\log x+\log y) \quad \text { and } \quad \log x=\sum_{n \geq 0} m_{n} x^{n+1}
$$

for computing the elements $\delta_{i}$ in $B P$ theory by the algorithm of Section 3.

Example 1 For $\delta_{1} \in B P^{*}(B \mathbf{Z} / 2)=B P^{*}[[z]] /([2](z))$ we have modulo $z^{8}$ :

$\delta_{1}=v_{1}^{2} z^{2}+\left(v_{1}^{3}+v_{2}\right) z^{3}+v_{1} z^{4}+\left(v_{1}^{6}+v_{1}^{3} v_{2}\right) z^{6}+\left(v_{1}^{4} v_{2}+v_{2}^{2}+v_{3}\right) z^{7}$.

Next we give some results of calculations in Morava $K$-theories, where the formulas are more tractable. In the following examples $\delta_{i}^{(k)}$ coincides with the coefficient at $\sigma_{p}^{i}$ in the expression for $\sigma_{k}$ from Corollary 5.4, $y=z^{p-1}$, and $\tilde{\delta}_{i}^{(k)}=k !(p-k) ! \delta_{i}^{(k)}$.

Example $2 p=3, s=2$

$\sigma_{1}=v_{2} y^{3} \sigma_{3}+v_{2} y^{4} x$.

$\sigma_{2}=2 v_{2}^{2} y^{3} \sigma_{3}^{4}+2 v_{2} y^{2} \sigma_{3}^{2}+v_{2} x^{2} y^{4}+2 y$.

Example $3 p=5, s=3$

Algebraic 63 Geometric Topology, Volume 3 (2003) 


$$
\begin{aligned}
& \sigma_{1}=v_{3} y^{25} \sigma_{5}{ }^{5}+v_{3} y^{30} \sigma_{5}+v_{3} y^{31} x \\
& \sigma_{2}=4 v_{3}{ }^{2} y^{25} \sigma_{5}{ }^{30}+4 v_{3}{ }^{2} y^{30} \sigma_{5}{ }^{26}+3 v_{3} y^{19} \sigma_{5}{ }^{10}+v_{3} y^{24} \sigma_{5}{ }^{6}+3 v_{3} y^{29} \sigma_{5}{ }^{2}+2 v_{3} y^{31} x^{2} \\
& \sigma_{3}=2 v_{3}{ }^{3} y^{25} \sigma_{5}{ }^{55}+2 v_{3}{ }^{3} y^{30} \sigma_{5}{ }^{51}+v_{3}{ }^{2} y^{19} \sigma_{5}{ }^{35}+2 v_{3}{ }^{2} y^{24} \sigma_{5}{ }^{31}+v_{3}{ }^{2} y^{29} \sigma_{5}{ }^{27}+ \\
& 2 v_{3} y^{13} \sigma_{5}{ }^{15}+v_{3} y^{18} \sigma_{5}{ }^{11}+v_{3} y^{23} \sigma_{5}{ }^{7}+2 v_{3} y^{28} \sigma_{5}{ }^{3}+2 v_{3} x^{3} y^{31} \\
& \sigma_{4}=4 v_{3}{ }^{4} y^{25} \sigma_{5}{ }^{80}+4 v_{3}{ }^{4} y^{30} \sigma_{5}{ }^{76}+4 v_{3}{ }^{3} y^{19} \sigma_{5}{ }^{60}+3 v_{3}{ }^{3} y^{24} \sigma_{5}{ }^{56}+4 v_{3}{ }^{3} y^{29} \sigma_{5}{ }^{52}+ \\
& 4 v_{3}{ }^{2} y^{13} \sigma_{5}{ }^{40}+2 v_{3}{ }^{2} y^{18} \sigma_{5}{ }^{36}+2 v_{3}{ }^{23} y_{5}{ }^{32}+4 v_{3}{ }^{2} y^{28} \sigma_{5}{ }^{28}+4 v_{3} y^{7} \sigma_{5}{ }^{20}+v_{3} y^{12} \sigma_{5}{ }^{16}+ \\
& 4 v_{3} y^{17} \sigma_{5}{ }^{12}+v_{3} y^{22} \sigma_{5}{ }^{8}+4 v_{3} y^{27} \sigma_{5}{ }^{4}+v_{3} x^{4} y^{31}+4 y .
\end{aligned}
$$

\section{$6 \quad$ Transfer and $K(s)^{*}\left(X_{h \Sigma_{p}}^{p}\right)$}

Let $X$ be a CW complex whose Morava $K$-theory $K(s)^{*}(X)$ is even dimensional and finitely generated as a module over $K(s)^{*}$.

In this section we study the transfer homomorphism in this more general context. We extend some results of Hopkins-Kuhn-Ravenel [10] to spaces. We consider the Atiyah-Hirzebruch-Serre (later abbreviated AHS) spectral sequence:

$$
E_{2}^{*, *}(\pi, X)=H^{*}\left(\pi ; K(s)^{*} X^{p}\right) \Rightarrow K(s)^{*}\left(X_{h \pi}^{p}\right) .
$$

By the Künneth isomorphism

$$
K(s)^{*} X^{p} \stackrel{\approx}{\longrightarrow}\left(K(s)^{*} X\right)^{\otimes p} .
$$

Then $K(s)^{*} X^{p}$ is a $\pi$ module where $\pi$ acts by permuting factors (see [10], Theorem 7.3).

An element $x \in K(s)^{*}(X)$ is called good if there is a finite cover $Y \rightarrow X$ together with an Euler class $y \in K(s)^{*}(Y)$ such that $x=\operatorname{Tr}^{*}(y)$ where $\operatorname{Tr}^{*}$ : $K(s)^{*}(Y) \rightarrow K(s)^{*}(X)$ is the transfer. The space $X$ is called good if $K(s)^{*}(X)$ is spanned over $K(s)^{*}$ by good elements.

Let $\gamma=\varphi^{*}(z)$, where

$$
\varphi: X_{h \pi}^{p} \rightarrow B \pi
$$

is the projection and let $\left\{x_{j}, j \in \mathcal{J}\right\}$ be a $K(s)^{*}$ basis for $K(s)^{*}(X)$. Hunton [12] has shown that if $K(s)^{*}(X)$ is concentrated in even dimensions then so is $K(s)^{*}\left(X_{h \pi}^{p}\right)$. We adopt the stronger hypothesis that $X$ is good and derive a stronger result, following the argument of [10] Theorem 7.3 for classifying spaces. 
Proposition 6.1 Let $X$ be a good space.

(i) As a $K(s)^{*}$ module $K(s)^{*}\left(X_{h \pi}^{p}\right)$ is free with basis

$$
\left\{\gamma^{i} \otimes\left(x_{j}\right)^{\otimes p} \mid 0 \leq i<p^{s}, j \in \mathcal{J}\right\}
$$

and

$$
\left\{\sum_{\left(i_{1}, i_{2}, \ldots, i_{p}\right)=I} 1 \otimes x_{i_{1}} \otimes x_{i_{2}} \otimes \cdots \otimes x_{i_{p}} \mid I \in \mathcal{P}_{p}\right\}
$$

where $I=\left\{\left(i_{1}, i_{2}, \ldots, i_{p}\right)\right\}$ runs over the set $\mathcal{P}_{p}$ of $\pi$-equivalence classes of $p$-tuples of indices $i_{j} \in \mathcal{J}$ at least two of which are not equal.

(ii) $X_{h \pi}^{p}$ is good.

Proof (i) By the Künneth isomorphism,

$$
K(s)^{*}(X)^{\otimes p}=F \oplus T
$$

as a $\pi$-module, where $F$ is free and $T$ is trivial. Explicitly a $K(s)^{*}$ basis for $T$ is $\left\{\left(x_{i}\right)^{\otimes p}, i \in \mathcal{J}\right\}$, while a $K(s)^{*}$ basis for $F$ is $\left\{x_{i_{1}} \otimes x_{i_{2}} \otimes \cdots \otimes x_{i_{p}}, i_{j} \in \mathcal{J}\right\}$ where not all the factors are equal. Then

$$
\begin{aligned}
& H^{*}(\pi ; F)=F^{\pi} \quad \text { if } \quad *=0 \\
& =0 \quad \text { if } \quad *>0
\end{aligned}
$$

and

$$
H^{*}(\pi ; T)=H^{*}(B \pi) \otimes T .
$$

Thus $E_{2}{ }^{0, *}(\pi, X)=K(s)^{*}\left(X_{h \pi}^{p}\right)^{\pi}=F^{\pi} \oplus T$.

To continue the proof we recall the covering projection

$$
\rho_{\pi}: E \pi \times X^{p} \rightarrow X_{h \pi}^{p},
$$

its associated transfer homorphism

$$
T r^{*}=T r_{\pi}^{*}: K(s)^{*}\left(X^{p}\right) \rightarrow K(s)^{*}\left(X_{h \pi}^{p}\right),
$$

and induced homomorphism

$$
\rho_{\pi}^{*}: K(s)^{*}\left(X_{h \pi}^{p}\right) \rightarrow K(s)^{*}\left(X^{p}\right) .
$$

Similar maps are defined for the group $\Sigma_{p}$. Then $\rho_{\pi}{ }^{*} \operatorname{Tr}^{*}=N$, where $N=N_{\pi}$ is the trace map.

Thus we have established the following lemma.

Algebraic 6 Geometric Topology, Volume 3 (2003) 
Lemma 6.2 If $y \in K(s)^{*}\left(X^{p}\right)$ is good then there exists a good element $z \in$ $K(s)^{*}\left(X_{h \pi}^{p}\right)$ such that $\rho_{\pi}^{*}(z)=N(y)$.

Lemma 6.3 If $x \in K(s)^{*}(X)$ is good then there is a good element $z \in$ $K(s)^{*}\left(X_{h \pi}^{p}\right)$ such that $\rho_{\pi}^{*}(z)=x^{\otimes p}$.

Proof By assumption there is a finite covering $f: Y \rightarrow X$ and an Euler class $e \in K(s)^{*}(Y)$ such that $x=T r^{*}(e)$. Now consider the covering

$$
\phi=f \times \cdots \times f: Y^{p} \rightarrow X^{p}
$$

which extends to a covering

$$
1 \times \phi: Y_{h \pi}^{p} \rightarrow X_{h \pi}^{p}
$$

and yields a map of coverings

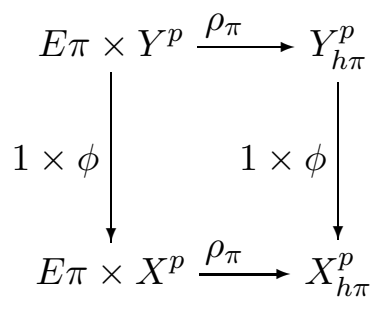

The class $e^{\otimes p}$ is an Euler class for $Y^{p}$. Since the transfer is natural and commutes with tensor products we have

$\rho_{\pi}^{*} \operatorname{Tr}^{*}\left(1 \otimes e^{\otimes p}\right)=\operatorname{Tr}^{*} \rho_{\pi}^{*}\left(1 \otimes e^{\otimes p}\right)=\operatorname{Tr}^{*}\left(e^{\otimes p}\right)=\operatorname{Tr}^{*}(e) \otimes \cdots \otimes \operatorname{Tr}^{*}(e)=x^{\otimes p}$.

Corollary 6.4 $E_{2}{ }^{0, *}(\pi, X)$ consists of permanent cycles which are good.

Thus as differential graded $K(s)^{*}$ modules, there is an isomorphism of spectral sequences

$$
\left(E_{r}^{*, *}(\pi, p t) \otimes_{K(s)^{*}} T\right) \oplus F^{\pi} \stackrel{\approx}{\underset{ }{\longrightarrow}} E_{r}^{*, *}(\pi, X) .
$$

Thus it follows that as a $K(s)^{*}$ algebra, $K(s)^{*}\left(X_{h \pi}^{p}\right)$ is generated by $K(s)^{*}(B \pi), T$, and $F^{\pi}$.

(ii) The proof of [10] Theorem 7.3 carries over. This completes the proof of Proposition 6.1.

Algebraic 63 Geometric Topology, Volume 3 (2003) 
Remarks (1) From the periodicity of the cohomology of a cyclic group [5] Proposition XII, 11.1, we have isomorphisms

$$
H^{t}\left(\pi ; K(s)^{*}\left(X^{p}\right)\right) \stackrel{\cdot z}{\longrightarrow} H^{t+2}\left(\pi ; K(s)^{*}\left(X^{p}\right)\right)
$$

for $t>0$ and

$$
H^{0}\left(\pi ; K(s)^{*}\left(X^{p}\right)\right) / \operatorname{Im}(N) \stackrel{\cdot z}{\longrightarrow} H^{2}\left(\pi ; K(s)^{*}\left(X^{p}\right)\right) .
$$

Thus multiplication by $z$ is also injective on $T$ at the $E_{2}$ term.

(2) $\rho_{\pi}{ }^{*} \operatorname{Tr}^{*}=N$, thus modulo $\operatorname{ker}\left(\rho_{\pi}{ }^{*}\right)$ we have

$$
\operatorname{Tr}^{*}\left(x_{i_{1}} \otimes x_{i_{2}} \otimes \cdots \otimes x_{i_{p}}\right)=\sum_{\sigma \in \pi} 1 \otimes x_{\sigma\left(i_{1}\right)} \otimes x_{\sigma\left(i_{2}\right)} \otimes \cdots \otimes x_{\sigma\left(i_{p}\right)} .
$$

Note that if the $i_{j}$ in (38) are equal, the right hand side is zero. However

$$
\operatorname{Tr}^{*}\left(x_{j}{ }^{\otimes p}\right)=1 \otimes x_{j}{ }^{\otimes p} \cdot \operatorname{Tr}^{*}(1) .
$$

We now turn to $K(s)^{*}\left(X_{h \Sigma_{p}}^{p}\right)$.

Let $c=\varphi^{*}(y)$ where $\varphi: E \Sigma_{p} \times_{\Sigma_{p}} X^{p} \rightarrow B \Sigma_{p}$ is the projection.

Proposition 6.5 Let $X$ be a good space. As a $K(s)^{*}$ module $K(s)^{*}\left(X_{h \Sigma_{p}}^{p}\right)$ is free with basis

$$
\left\{c^{i} \otimes\left(x_{j}\right)^{\otimes p} \mid 0 \leq i<m_{s}, j \in \mathcal{J}\right\}
$$

and

$$
\left\{\sum_{\left(i_{1}, i_{2}, \ldots, i_{p}\right)=I} 1 \otimes x_{i_{1}} \otimes x_{i_{2}} \otimes \cdots \otimes x_{i_{p}} \mid I \in \mathcal{E}_{p}\right\}
$$

where $I=\left\{\left(i_{1}, i_{2}, \ldots, i_{p}\right)\right\}$ runs over the set $\mathcal{E}_{p}$ of $\Sigma_{p}$-equivalence classes of $p$-tuples of indices $i_{j} \in \mathcal{J}$ at least two of which are not equal.

Proof Since $|W|$ is prime to $p$, the result follows from the AHS spectral sequence, as in the proof of Proposition 6.1.

\section{Applications}

$7.1 \pi \imath\left(\mathbf{Z} / p^{n}\right)$

We now turn to $G_{n}=\pi \imath\left(\mathbf{Z} / p^{n}\right)$ where $\pi=\mathbf{Z} / p$. Then $B G_{n}=X_{h \pi}^{p}$ for $X=B \mathbf{Z} / p^{n}$. Consider the AHS spectral sequence for

$$
B\left(\mathbf{Z} / p^{n}\right)^{p} \rightarrow B G_{n} \stackrel{\varphi}{\rightarrow} B \pi .
$$

Algebraic 83 Geometric Topology, Volume 3 (2003) 
Then

$$
E_{2}^{p, q}=H^{*}\left(\pi ; K^{*}(s)\left(B\left(\mathbf{Z} / p^{n}\right)^{p}\right)\right)
$$

where

$$
K^{*}(s)\left(B\left(\mathbf{Z} / p^{n}\right)^{p}\right)=\left(K(s)^{*}[z] /\left(z^{p^{n s}}\right)\right)^{\otimes p}=F \oplus T,
$$

where $F$ and $T$ as in (36) above are free (resp. trivial) $\pi$ modules.

Let $\gamma=\varphi^{*}(z)$ where $K(s)^{*}(B \pi)=K(s)^{*}[z] /\left(z^{p^{s}}\right)$ as above.

Proposition 7.1 As a $K(s)^{*}$ module $K(s)^{*}\left(B G_{n}\right)$ is free with basis

$$
\left\{\gamma^{i} \otimes\left(z^{j}\right)^{\otimes p} \quad 0 \leq i<p^{s}, 0 \leq j<p^{n s}\right\}
$$

and

$$
\left\{\sum_{\left(i_{1}, i_{2}, \ldots, i_{p}\right)=I} 1 \otimes z^{i_{1}} \otimes z^{i_{2}} \otimes \cdots \otimes z^{i_{p}} \quad \mid I \in \mathcal{P}_{p}(n)\right\},
$$

where $I=\left\{\left(i_{1}, i_{2}, \ldots, i_{p}\right)\right\}$ runs over the set $\mathcal{P}_{p}(n)$ of $\pi$-equivalence classes of $p$-tuples of integers $\left\{0 \leq i_{j}<p^{n s}\right\}$ at least two of which are not equal.

Proof This spectral sequence computation is exactly analogous to that of Proposition 6.1.

Remarks (i) For $X=\mathbf{C} P^{\infty}$, Proposition 7.1 gives another derivation of $K(s)^{*}\left(X_{h \pi}^{p}\right)$. Since $\mathbf{C} P_{p}^{\infty \wedge}=\left[\operatorname{colim}_{n} B\left(\mathbf{Z} / p^{n}\right)\right]_{p}^{\wedge}$, we have $K(s)^{*}\left(X_{h \pi}^{p}\right)=$ $\lim _{n} K(s)^{*}\left(B G_{n}\right)$.

(ii) $G_{n}$ is good for $K(s)^{*}$ by [10] Theorem 7.3.

By analogy with Section 6 we have the following:

Lemma 7.2 (i) $\operatorname{Im}\left(T r^{*}\right) \cdot \gamma=0$.

(ii) $\operatorname{Tr}^{*}(1)=v_{s} \gamma^{p^{s}-1}$.

(iii) If $y \in T$ then $\operatorname{Tr}^{*}(y)=y \cdot \operatorname{Tr}^{*}(1)$.

Finally we consider the case $p=2$ where $c=\gamma$. If $n=1, G_{n} \approx D_{8}$, the dihedral group of order 8; the rings $K(s)^{*}\left(B D_{2^{k}}\right)$ were determined by Schuster $[20],[21]$. In general we have a partial result:

Proposition 7.3 Let $p=2$. As a $K(s)^{*}(B \pi)$ algebra, $K(s)^{*}\left(B G_{n}\right)$ is generated by $\tilde{c}_{1}, c_{2}$ subject to the relation $\tilde{c}_{1} \cdot c=0$ and the relations $\tilde{c}_{1}^{2^{n s}}=c_{2}{ }^{2^{n s}}=0$ modulo terms divisible by $c$. 
Proof $\tilde{c}_{1} \cdot c=0$ by Lemma 7.2. The other relations hold in the $E_{\infty}$ term of the spectral sequence. The only possible extensions are those on the fiber, involving $\tilde{c}_{1}, c_{2}$.

Similar results hold for $\Sigma_{p} \prec \mathbf{Z} / p$ and $\Sigma_{p} \prec \Sigma_{p}$ for $p$ odd.

7.2 $p$-groups with cyclic subgroup of index $p$.

In this section we consider the class of $p$-groups with a (necessarily normal) cyclic subgroup of index $p$. It is known [3], Theorem 4.1, Chapter IV, that every $p$-group of this form is isomorphic to one of the groups:

(a) $\mathbf{Z} / q\left(q=p^{n}, n \geq 1\right)$.

(b) $\mathbf{Z} / q \times \mathbf{Z} / p\left(q=p^{n}, n \geq 1\right)$.

(c) $\mathbf{Z} / q \rtimes \mathbf{Z} / p\left(q=p^{n}, n \geq 2\right)$, where the canonical generator of $\mathbf{Z} / p$ acts on $\mathbf{Z} / q$ as multiplication by $1+p^{n-1}$. This group is called the modular group if $p \geq 3$ and the quasi-dihedral group if $p=2, n \geq 4$.

For $p=2$ there are three additional families.

(d) Dihedral 2-groups $D_{2 m}=\mathbf{Z} / m \rtimes \mathbf{Z} / 2,(m \geq 2)$, where the generator of $\mathbf{Z} / 2$ acts on $\mathbf{Z} / m$ as multiplication by -1 . If $m=2^{n}, D_{2 m}$ is a 2-group. Note that $D_{4}$ belongs to (b) and $D_{8}$ belongs to (c).

(e) Generalized quaternion 2-groups. Let $\mathbf{H}$ be the algebra of quaternions $\mathbf{R} \oplus \mathbf{R} i \oplus \mathbf{R} j \oplus \mathbf{R} k$. For $m \geq 2$ the generalized quaternion group $Q_{4 m}$ is defined as the subgroup of the multiplicative group $\mathbf{H}^{*}$ generated by $x=e^{\pi i / m}$ and $y=j . \mathbf{Z} / 2 m$ generated by $x$ is normal and has index 2 . If $m$ is a power of $2, Q_{4 m}$ is a 2 -group. In the extension $0 \rightarrow \mathbf{Z} / 2 m \rightarrow Q_{4 m} \rightarrow \mathbf{Z} / 2 \rightarrow 0$ the generator of $\mathbf{Z} / 2$ acts on $\mathbf{Z} / 2 m$ as -1 . In particular $Q_{8}$ is the group of quaternions $\{ \pm 1, \pm i, \pm j, \pm k\}$.

(f) Semi-dihedral groups. $\mathbf{Z} / q \rtimes \mathbf{Z} / 2\left(q=p^{n}, n \geq 3\right)$, where the generator of $\mathbf{Z} / 2$ acts on $\mathbf{Z} / q$ as multiplication by $-1+2^{n-1}$.

Consider now the task of computing the stable Euler class, $\operatorname{Tr}_{G}^{*}(1)$, for the universal $G$-covering $E G \rightarrow B G$.

For the case (a) there is the well known formula of Quillen (1)

$$
\operatorname{Tr}_{\mathbf{Z} / q}^{*}(1)=[q]_{F}(z) / z
$$

in $M U^{*}(B \mathbf{Z} / q)=M U^{*}[[z]] /\left([q]_{F}(z)\right)$. 
For the case (b) the answer follows from transfer property (ii): $\operatorname{Tr}_{G}=\operatorname{Tr}_{\mathbf{Z} / q} \wedge$ $\operatorname{Tr}_{\mathbf{Z} / p}$

In cases (d),(e) and (f) $\operatorname{Tr}_{G}^{*}$ is the composition of two transfers $\operatorname{Tr}_{\mathbf{Z} / q}^{*}$ and $\operatorname{Tr}_{C, G}^{*}: M U^{*}(B C) \rightarrow M U^{*}(B G)$, where $C$ is the corresponding cyclic subgroup. So we have to compute $\operatorname{Tr}_{C, G}^{*}\left(z^{i}\right), i \geq 1$ and we can apply our results for $B \mathbf{Z} / 2$ 乙 $U(1)$, namely Remark 3.7.

Similarly for the case (c), $\operatorname{Tr}_{G}$ is the composition $\operatorname{Tr}_{\mathbf{Z} / q, G} \operatorname{Tr}_{\mathbf{Z} / q}$ and we can apply Corollary 3.6.

This task is trivial for wreath products $\mathbf{Z} / p \nmid \mathbf{Z} / p^{n}$ since $\operatorname{Tr}_{\mathbf{Z} / p^{n}}^{*}(1)$ is symmetric in $z_{1}, \ldots, z_{p}$ in the ring

$$
M U^{*}\left(\left(B \mathbf{Z} / p^{n}\right)^{p}\right)=M U^{*}\left[\left[z_{1}, \ldots, z_{p}\right]\right] /\left(\left[p^{n}\right]\left(z_{1}\right), \ldots,\left[p^{n}\right]\left(z_{p}\right)\right)
$$

and hence invariant under the $\mathbf{Z} / p$ action. So in this case we need only Quillen's formula.

Finally we note that if $G$ is the modular group of case (c), Brunetti [4] has completely computed the ring $K(s)^{*}(B G)$. The relations are quite simple but the generators are technically complicated. In a future paper we plan to use transferred Chern classes to give a more natural presentation.

\subsection{Other examples}

Consider the semi-direct products $G=(\mathbf{Z} / p)^{n} \rtimes \mathbf{Z} / p$ where the generator $\alpha$ of $\mathbf{Z} / p$ acts on $H_{n}=\mathbf{Z} / p[T] /\left(T^{n}\right)$ by $1-\alpha=T, 1 \leq n \leq p$. Then every $\mathbf{Z} / p[\mathbf{Z} / p]$-module is a direct sum of the modules $H_{n}$. As shown by Yagita [24] and Kriz [14], these semi-direct products are good in the sense of HopkinsKuhn-Ravenel.

We recall

$$
K(s)^{*}\left(B(\mathbf{Z} / p)^{n}\right)=K(s)^{*}\left[\left[z_{1}, \ldots, z_{n}\right]\right] /\left(z_{i}^{p^{s}}\right),
$$

where $z_{i}$ is the Euler class of a faithful complex line bundle $\theta_{i}$ on the $i$-th factor. Then $\mathbf{Z} / p$ acts on $K(s)^{*}\left[z_{1}, \ldots, z_{n}\right] /\left(z_{i}^{p^{s}}\right)$ by

$$
\alpha: z_{i} \rightarrow F_{K(s)}\left(z_{i}, z_{i+1}\right), \quad z_{n+1}:=0,
$$

where $F_{K(s)}$ denotes the formal group law for Morava $K$-theory.

Our aim is to show how to compute the stable Euler classes in terms of characteristic classes and the formal group law.

The transfer $T r^{*}: K(s)^{*} E G \rightarrow K(s)^{*} B G$ is the composition of two transfers

$$
\operatorname{Tr}_{1}^{*}: K(s)^{*} E\left((\mathbf{Z} / p)^{n}\right) \rightarrow K(s)^{*} B\left((\mathbf{Z} / p)^{n}\right)
$$


and

$$
\operatorname{Tr}_{2}^{*}: K(s)^{*} B\left((\mathbf{Z} / p)^{n}\right) \rightarrow K(s)^{*} B G .
$$

Recall also that

$$
\operatorname{Tr}_{1}^{*}(1)=z_{1}^{p^{s}-1} \cdots z_{n}^{p^{s}-1} .
$$

It is easy to see that in $K(s)^{*}\left((B \mathbf{Z} / p)^{n}\right)$ we have

$$
e^{p^{s}-1}\left(\alpha^{i_{1}}\left(\theta_{1}\right)\right) \cdots e^{p^{s}-1}\left(\alpha^{i_{n}}\left(\theta_{1}\right)\right)=z_{1}^{p^{s}-1} \cdots z_{n}^{p^{s}-1},
$$

where $e$ is the Euler class and $1 \leq i_{1}<\cdots<i_{n} \leq p$. Then recall the elements $\omega_{n}$ from Theorem 3.1 and let $\omega_{n}(l)$ be the sum of the same monomials after raising to the power $l$. Since $\omega_{n}(l)$ consist of $p^{-1}\left(\begin{array}{l}p \\ n\end{array}\right)$ summands and $p^{-1}\left(\begin{array}{l}p \\ n\end{array}\right)=(-1)^{n} / n \bmod p$, we have that in $K(s)^{*}\left((B Z / p)^{n}\right)$

$$
\eta_{\pi}^{*}\left(\omega_{n}\left(p^{s}-1\right)\right)=(-1)^{n} z_{1}^{p^{s}-1} \cdots z_{n}^{p^{s}-1} / n,
$$

where the map $\eta_{\pi}$, defined in Section 2 , sends $\xi_{i}=t^{i-1} \xi_{1}$ to $\alpha^{i-1} \theta_{1}$. Hence

$$
\begin{gathered}
\operatorname{Tr}_{G}^{*}(1)=\operatorname{Tr}_{2}^{*}\left(\operatorname{Tr}_{1}^{*}(1)\right)=\operatorname{Tr}_{2}^{*}\left((-1)^{n} n \eta_{\pi}^{*}\left(\omega_{n}\left(p^{s}-1\right)\right)\right)= \\
(-1)^{n} n \operatorname{Tr}_{2}^{*}\left(\eta_{\pi}^{*}\left(\omega_{n}\left(p^{s}-1\right)\right)\right),
\end{gathered}
$$

and we have to apply Corollary 3.6.

\section{References}

[1] J.F. Adams : Infinite Loop Spaces. Annals of Mathematics Studies, Princeton University Press, Princeton, (1978).

[2] M.F. Atiyah : Characters and cohomology of finite groups, Publ. Math. of the I.H.E.S. 9, (1961), 23-64.

[3] K.S. Brown : Cohomology of groups, Grad. Texts in Math. 87, Springer, (1982).

[4] M. Brunetti :Morava $K$-theory of $p$-groups with cyclic maximal subgroups and other related p-groups, $K$-Theory 24, (2001), 385-395.

[5] H. Cartan and S. Eilenberg : Homological Algebra, Princeton Math. Series no. 19, Princeton University Press, Princeton, (1956).

[6] T. tom Dieck: Transformation groups and representation theory, Lecture Notes in Math. 766, (1979).

[7] A. Dold : The fixed point transfer of fibre-preserving maps, Math. Zeit. 148, (1976), 215-244.

[8] M. Hazewinkel : Constructing formal groups III, Applications to complex cobordism and Brown-Peterson cohomology, J. Pure Appl. Algebra 10, (1977/78), $1-18$.

Algebraic 83 Geometric Topology, Volume 3 (2003) 
[9] F. Hirzebruch, T. Berger, and R. Jung : Manifolds and Modular Forms Aspects of Mathematics, E20, Friedr. Vieweg \& Sohn, Braunschweig, (1992).

[10] M. Hopkins, N. Kuhn, and D. Ravenel : Generalized group characters and complex oriented cohomology theories, J. Amer. Math. Soc. 13 3(2000), 553-594.

[11] J. Hunton : The Morava $K$-theories of wreath products, Math. Proc. Camb. Phil. Soc. 107, (1990), 309-318.

[12] J. Hunton: The complex oriented cohomology of extended powers, Ann. Inst. Fourier, Grenoble 48, 2(1998), 517-534.

[13] D.S. Kahn, S.B. Priddy : Applications of the transfer to stable homotopy theory, Bull Amer. Math. Soc. 78, (1972), 981-987.

[14] I. Kriz : Morava K -theory of classifying spaces: Some calculations, Topology 36, (1997), 1247-1273.

[15] J. McClure and V. Snaith :On the $K$-theory of the extended power construction, Proc. Camb. Phil. Soc. 92, (1982), 263-274.

[16] D. Quillen : Elementary proofs of some results of cobordism theory using Steenrod operations, Advances in Math. 7, (1971), 29-56.

[17] D. Ravenel : Complex cobordism and Stable Homotopy Groups of Spheres, Academic Press, (1986).

[18] D. Ravenel and S. Wilson: The Morava K-theories of Eilenbeg-MacLane spaces and the Conner-Floyd conjecture, Amer. J. of Math. 102,4(1980), 691-748.

[19] H. Sadofsky : The root invariant and $v_{i}$-periodic families, Topology 31 (1991), 65-111.

[20] B. Schuster :On the Morava K-theory of some finite 2-groups, Math. Proc. Camb. Phil. Soc. 121 (1997), 7-13.

[21] B. Schuster and N. Yagita :Morava $K$-theory of extraspecial 2 -groups, preprint.

[22] M. Tezuka and N. Yagita :Cohomology of finite groups and Brown-Peterson cohomology, Algebraic Topology Arcata 1986, Springer LNM 1370 (1989), 396408.

[23] U. Würgler : Commutative ring spectra in characteristic 2, Comm. Math. Helv. 61, (1986), 33-45.

[24] N. Yagita :Note on BP-theory for extensions of cyclic groups by elementary abelian p-groups, Kodai Math. J. 20 2(1997), 79-84.

Razmadze Mathematical Institute, Tbilisi 380093, Republic of Georgia and

Department of Mathematics, Northwestern University, Evanston, IL 60208, USA

Email: maxo@rmi.acnet.ge, priddy@math.northwestern.edu

Received: 26 September 2002 Revised: 12 May 2003 